\title{
Finite volume expectation values in the sine-Gordon model
}

\author{
Árpád Hegedüs \\ Wigner Research Centre for Physics, \\ Budapest 114, P.O. Box 49, H-1525 Hungary \\ E-mail: hegedus.arpad@wigner.mta.hu
}

ABSTRACT: Using the fermionic basis discovered in the 6-vertex model, we derive exact formulas for the expectation values of local operators of the sine-Gordon theory in any eigenstate of the Hamiltonian. We tested our formulas in the pure multi-soliton sector of the theory. In the ultraviolet limit, we checked our results against Liouville 3-point functions, while in the infrared limit, we evaluated our formulas in the semi-classical limit and compared them up to 2-particle contributions against the semi-classical limit of the previously conjectured LeClair-Mussardo type formula. Complete agreement was found in both cases.

Keywords: Bethe Ansatz, Integrable Field Theories

ARXIV EPRINT: 1909.08467 


\section{Contents}

1 Introduction 1

2 Sine-Gordon model as a perturbed conformal field theory 2

2.1 Perturbed Liouville CFT formulation 3

2.2 Compactified free boson description 3

3 Integral equations for the spectrum $\quad 4$

4 The function $\omega$ for excited states $\quad 8$

5 Formulas for the expectation values $\quad 11$

$6 \quad$ Large volume checks $\quad \mathbf{1 3}$

6.1 Compatibility check of the formulas (5.9) and (5.12) 13

6.2 Connected diagonal form-factors of $\Phi_{4 \frac{1-\nu}{\nu}}(0) \quad 16$

6.3 Classical limit of connected diagonal form factors 21

$\begin{array}{lll}7 & \text { Small volume checks } & \mathbf{2 5}\end{array}$

7.1 The case of primaries $\Phi_{2 \frac{1-\nu}{\nu}}(0)$ and $\Phi_{4 \frac{1-\nu}{\nu}}(0) \quad 28$

$\begin{array}{ll}7.2 \text { Expectation values of descendant fields } & \nu \\ & \end{array}$

$\begin{array}{lll}\text { 7.2.1 The case of }\left\langle\mathbf{1}_{-2} \Phi_{2 \frac{1-\nu}{\nu}}\right\rangle & 29\end{array}$

7.2.2 Expectation values of the descendants of the unity 31

8 Summary and conclusions 33

\section{Introduction}

The knowledge of finite volume form-factors of integrable quantum field theories became important in string theory and in condensed matter applications, as well. In string-theory, they arise in the AdS/CFT correspondence when heavy-heavy-light 3-point functions are considered [1-5]. In condensed matter physics the finite volume form-factors are necessary to represent correlation functions for describing various quasi 1-dimensional condensed matter systems [6].

So far two basic approaches have been developed to compute finite volume matrix elements of local operators in an integrable quantum field theory. In the first approach $[7,8]$, the finite-volume form-factors are represented as a large volume series in terms of the infinite volume form-factors of the theory. In this approach the polynomial in volume corrections are given by the Bethe-Yang quantizations of the rapidities, while the exponentially 
small in volume Lüscher corrections come from contributions of virtual particles propagating around the compact dimension [9-11]. In a diagonally scattering theory, the diagonal matrix elements of local operators can be computed by means of the LeClair-Mussardo series [19-21], provided the connected diagonal form-factors of the operator under consideration are known. In a non-diagonally scattering theory, so far the availability of such a series representation is restricted to the sine-Gordon model [24, 25].

The second approach, which works well for non-diagonally scattering theories, as well, is based on some integrable lattice regularization of the model under consideration. The results of [13] for the sine-Gordon model made it possible to conjecture exact formulas for the finite volume diagonal matrix elements of the sinh-Gordon model $[15,16]$, as well. The main advantage of the lattice approach is that, it provides exact formulas for the (specific ratios of) finite-volume form-factors. From this approach, the corresponding LeClair-Mussardo series can be obtained by the large volume series expansion of the formulas. In [13] the finite volume ground state expectation values of the exponential fields and their descendants have been determined in the sine-Gordon model. In this paper we extend these results to get formulas for the expectation values of local operators in any excited state of the theory.

The outline of the paper is as follows: in section 2, we formulate the sine-Gordon model as perturbed conformal field theory. In section 3, we review the equations governing the finite volume spectrum of the theory. In section 4, we derive equations for the function $\omega$, which is the fundamental building block of the expectation value formulas of local operators. In section 5, we recall from [13], how the expectation values of the exponential fields and their descendants are built up from the function $\omega$. In section 6 , we perform the large volume checks of our formulas for the expectation values. The most important of them is a comparison to the classical limit of the previously conjectured [24] LeClair-Mussardo type large volume series representation. Section 7, contains the ultraviolet tests of our formulas. Finally, the paper is closed by our conclusions.

\section{Sine-Gordon model as a perturbed conformal field theory}

In this section, we recall the perturbed conformal field theory (PCFT) descriptions of the sine-Gordon model defined by the Euclidean action:

$$
\mathcal{A}_{\mathrm{SG}}=\int\left\{\frac{1}{4 \pi} \partial_{z} \varphi(z, \bar{z}) \partial_{\bar{z}} \varphi(z, \bar{z})+\frac{2 \mu^{2}}{\sin \pi \beta^{2}} \cos (\beta \varphi(z, \bar{z}))\right\} \frac{i d z \wedge d \bar{z}}{2},
$$

where $z=x+i y$ and $\bar{z}=x-i y$, with $x, y$ being the coordinates of the Euclidean space-time.

In the paper, we will use two perturbed conformal field theory formulations of this model. The first one is, when it is considered as a perturbed complex Liouville CFT, and the second one is when it is described as a perturbed $c=1$ compactified boson.

In the original paper [13], the formulation with complex Liouville CFT is used. Nevertheless, it turned out from the detailed [37-39] UV analysis of the finite volume spectrum, that all eigenstates of the Hamiltonian are in one to one correspondence to the operators of the $c=1$ modular invariant compactified boson CFT. Thus, we find eligible to describe the 
finite volume form factors of the operators corresponding to the $c=1$ PCFT description of the model.

In the later sections, we will see that in some sense, this set of operators plays a special role among the operators of the complex Liouville-theory. Namely, in the exact description of finite-volume form-factors [13], only the ratios of diagonal form-factors can be computed. But, for the operators corresponding to the $c=1 \mathrm{CFT}$, the explicit expectation values themselves can be computed exactly, and not only their ratios.

\subsection{Perturbed Liouville CFT formulation}

In [13], the sine-Gordon model is considered as a perturbed complex Liouville theory:

$$
\mathcal{A}_{\mathrm{SG}}=\mathcal{A}_{L}+\frac{\mu^{2}}{\sin \pi \beta^{2}} \int e^{-i \beta \varphi(z, \bar{z})} \frac{i d z \wedge d \bar{z}}{2},
$$

where $\mathcal{A}_{L}$ denotes the action of the complex Liouville CFT:

$$
\mathcal{A}_{L}=\int\left\{\frac{1}{4 \pi} \partial_{z} \varphi(z, \bar{z}) \partial_{\bar{z}} \varphi(z, \bar{z})+\frac{\mu^{2}}{\sin \pi \beta^{2}} e^{i \beta \varphi(z, \bar{z})}\right\} \frac{i d z \wedge d \bar{z}}{2} .
$$

The central charge of the CFT is

$$
c_{L}=1-6 \frac{\nu^{2}}{1-\nu}, \quad \nu=1-\beta^{2},
$$

where we introduced the parameter $\nu=1-\beta^{2}$ in order to fit to the notations of ref. [13]. We just mention, that $0<\nu<1$ is the range of the parameter, such that the ranges $\frac{1}{2}<\nu<1$, and $0<\nu<\frac{1}{2}$ correspond to the attractive and repulsive regimes of the model. The primary fields are labeled by the real continuous parameter $\alpha$ :

$$
\Phi_{\alpha}(z, \bar{z})=e^{\frac{i \alpha \beta \nu}{2(1-\nu)} \varphi(z, \bar{z})}
$$

and have scaling dimensions $2 \Delta_{\alpha}$ with:

$$
\Delta_{\alpha}=\frac{\nu^{2}}{4(1-\nu)} \alpha(\alpha-2) .
$$

Primary fields (2.5) and their descendants span the basis in the space of operators of the theory.

\subsection{Compactified free boson description}

The sine-Gordon theory can be formulated as the perturbation of a free compactified boson CFT. Now, the whole potential term of (2.1) plays the role of the perturbation:

$$
\mathcal{A}_{\mathrm{SG}}=\mathcal{A}_{B}+\frac{2 \mu^{2}}{\sin \pi \beta^{2}} \int \cos (\beta \varphi(z, \bar{z})) \frac{i d z \wedge d \bar{z}}{2},
$$

where $\mathcal{A}_{B}$ denotes the action of the free boson compactified on a circle of radius $R=\frac{1}{\beta}$ :

$$
\mathcal{A}_{B}=\int \frac{1}{4 \pi} \partial_{z} \varphi(z, \bar{z}) \partial_{\bar{z}} \varphi(z, \bar{z}) \frac{i d z \wedge d \bar{z}}{2} .
$$


The primary states of this CFT are created by the vertex operators $V_{n, m}(z, \bar{z})$ which are labeled by two quantum numbers $n \in \mathbb{R}$ and $m \in \mathbb{Z}$. Their left and right conformal dimensions are given by:

$$
\Delta_{n, m}^{ \pm}=\left(\frac{n}{R} \pm \frac{1}{4} m R\right)^{2}
$$

Here $n$ is the momentum quantum number, and $m$ is the winding number or topological charge. The requirement of the locality of the operator product algebra of the CFT imposes further severe restrictions on the possible values of the pair of quantum numbers $(n, m)$. It turns out [54], that only a bosonic and a fermionic maximal subalgebras of the vertex operators $V_{n, m}(z, \bar{z})$ are allowed. The bosonic subalgebra is characterized by the quantum numbers $\{n \in \mathbb{Z}, m \in \mathbb{Z}\}$. It corresponds to the modular invariant partition function and this CFT describes the UV limit of the sine-Gordon model [54].

In the fermionic subalgebra the allowed set of quantum numbers is given by $\{n \in$ $\mathbb{Z}, m \in 2 \mathbb{Z}\} \cup\left\{n \in \mathbb{Z}+\frac{1}{2}, m \in 2 \mathbb{Z}+1\right\}$. It corresponds to a $\Gamma_{2}$ invariant partition function and this CFT describes the UV limit of the massive-Thirring model [54].

The perturbing term in the action (2.7) is given in terms of these vertex operators as follows:

$$
\mathcal{A}_{\text {pert }}=\frac{\mu^{2}}{\sin \pi \beta^{2}} \int \frac{i d z \wedge d \bar{z}}{2}\left(V_{1,0}(z, \bar{z})+V_{-1,0}(z, \bar{z})\right)
$$

In this paper we will mostly focus on computing the diagonal matrix elements of the primaries and their descendants belonging to the bosonic subalgebra of the $c=1 \mathrm{CFT}$.

Diagonal matrix elements are non-zero only in the zero winding number sector $(m=0)$, thus our primary goal is to derive formulas for the diagonal matrix elements of the vertex operators $V_{n, 0}(z, \bar{z})$, and of their descendants with $n \in \mathbb{Z}$. In the Liouville formulation they correspond to the primaries $\Phi_{2 n \frac{1-\nu}{\nu}}(z, \bar{z})$ and their descendants.

\section{Integral equations for the spectrum}

In this section, we summarize the nonlinear integral equations (NLIE) governing the finite volume spectrum of the sine-Gordon theory.

Originally, the equations were derived from an inhomogeneous 6-vertex model with, which serves as an integrable light-cone lattice regularization [30] of the sine-Gordon model. Later, the same type of equations were obtained from an approriate lattice regularization for two coupled quantum KdV equations [41]-[43].

In the series of papers [32, 33]-[39], the starting point of the derivation of the equations is the Bethe-Ansatz solution of the inhomogeneous 6-vertex model, since it has been shown, at the level of the spectrum in refs. [26]-[29], and later at field theory level in [30], that the continuum limit of the 6 -vertex model with appropriately chosen alternating inhomogeneities is the massive Thirring model. The equivalence between the sine-Gordon and massive Thirring models [53, 54] allows one to consider the 6-vertex model as an appropriate lattice regularization for the sine-Gordon theory, as well. 
The first version of the NLIE was derived in [31], to obtain an exact description for the finite size dependence of the largest einevalue of the transfer matrix of the homogeneous 6 -vertex model. In this paper, the method of deriving the equations was based on TQrelations and the analyticity properties of the largest einevalue of the transfer matrix and of Baxter's Q-function in the complex plane of the spectral parameter.

Later, in [32-34] the same type of equations were derived for the ground state of the 6 -vertex model with alternating inhomogeneities, by a completely different method. In these papers, the contributions of the Bethe-roots condensated along the real axis of the complex plane, are summed up by a special contour integral containing the countingfunction of the Bethe-Ansatz solvable lattice model. Taking the continuum limit of the equations according to the prescription of [30], non-linear integral equations governing the finite volume dependence of the ground state energy of the sine-Gordon model was obtained [32-34].

In [35], it was recognized, that if holes are introduced into the sea of real Bethe-roots, then the contributions of these holes can also be taken into account exactly in the equations, and the new equations describe the energies of the pure soliton excited states of the model.

In [36], the configurations containing also complex Bethe-roots were considered, and the non-linear integral equations for the full finite volume spectrum of the sine-Gordon model, was obtained.

Nevertheless, the form of the NLIE of [36] was to be refined, since it didn't give the correct quantization rules of [35] for the pure soliton states. In papers [37, 38], it was recognized, that not all eigenstates of the 6-vertex model's transfer matrix correspond to physical states of the continuum quantum field theory (QFT), and to get the correct quantization conditions corresponding to the QFT, certain selection rules must be imposed. These rules could have been determined by the careful ultraviolet and Truncated Conformal Space Approach analysis of the model [37-39], making it possible to obtain the, in all respect correct form, of the non-linear integral equations governing the finite volume energies of the sine-Gordon and massive Thirring models.

The detailed derivation of the non-linear integral equations for the finite volume spectrum of the sine-Gordon model, can be found in [40]. Here, we just write down the final form of the equations for the continuum case.

The unknown-function of the nonlinear integral equations (NLIE) is the countingfunction of the 6 -vertex model. ${ }^{1}$ The counting-function $Z(\lambda)$ is an $i \pi$ periodic function. In the fundamental regime: $|\operatorname{Im}(\lambda)|<\min (\pi \nu, \pi(1-\nu))$, it satisfies the equations as follows [39];

$$
\begin{aligned}
& Z(\lambda)=\ell \sinh \frac{\lambda}{\nu}+g\left(\lambda \mid\left\{h_{j}\right\},\left\{\lambda_{j}\right\},\left\{y_{j}\right\}\right)- \\
& \int_{-\infty}^{\infty} \frac{d \lambda^{\prime}}{i} R\left(\lambda-\lambda^{\prime}-i 0 \mid 0\right) L_{+}\left(\lambda^{\prime}+i 0\right)+\int_{-\infty}^{\infty} \frac{d \lambda^{\prime}}{i} R\left(\lambda-\lambda^{\prime}+i 0 \mid 0\right) L_{-}\left(\lambda^{\prime}-i 0\right),
\end{aligned}
$$

\footnotetext{
${ }^{1}$ To be more precise, what we call counting-function in the sequel, is the continuum-limit of the countingfunction of the 6 -vertex model.
} 
where $\ell=\mathcal{M} L$ with $\mathcal{M}$ and $L$ being the soliton-mass and the size of the compactified dimension,

$$
\begin{aligned}
L_{ \pm}(\lambda)= & \ln \left(1+(-1)^{\delta} e^{ \pm i Z(\lambda)}\right), \quad \delta \in\{0,1\} \\
g\left(\lambda \mid\left\{h_{j}\right\},\left\{\lambda_{j}\right\},\left\{y_{j}\right\}\right)= & -2 \pi \sum_{j=1}^{m_{H}} \chi_{R}\left(\lambda-h_{j}\right)+2 \pi \sum_{j=1}^{m_{C}} \chi_{R}\left(\lambda-c_{j}\right)+2 \pi \sum_{j=1}^{m_{W}} \chi_{R, I I}\left(\lambda-w_{j}\right)+ \\
& 2 \pi \sum_{j=1}^{m_{S}}\left(\chi_{R}\left(\lambda-y_{j}-i 0\right)+\chi_{R}\left(\lambda-y_{j}+i 0\right)\right) .
\end{aligned}
$$

The functions $R$ and $\chi_{R}$ entering the equations are of the form:

$$
\begin{aligned}
& R(\lambda \mid \alpha)=\int_{-\infty}^{\infty} \frac{d \omega}{2 \pi} e^{i \omega \lambda} \tilde{R}(\omega \mid \alpha), \quad \tilde{R}(\omega \mid \alpha)=\frac{1}{2} \frac{\sinh \left[\frac{\pi \omega}{2}(2 \nu-1)-\frac{i \pi \alpha}{2}\right]}{\cosh \frac{\pi \nu \omega}{2} \sinh \left[\frac{\pi \omega}{2}(1-\nu)+\frac{i \pi \alpha}{2}\right]}, \\
& \chi_{R}(\lambda)=\int_{-\infty}^{\infty} \frac{d \omega}{2 \pi} \frac{\sinh (\omega \lambda)}{\omega} \tilde{R}(\omega \mid 0) .
\end{aligned}
$$

The label $I I$ on any function means second determination with the definition as follows [36]:

$$
f_{I I}(\lambda)=\left\{\begin{aligned}
f(\lambda)+f(\lambda-i \pi \nu \operatorname{sign}(\operatorname{Im} \lambda)), & 0<\nu<\frac{1}{2} \\
f(\lambda)-f(\lambda-i \pi(1-\nu) \operatorname{sign}(\operatorname{Im} \lambda)), & \frac{1}{2}<\nu<1 .
\end{aligned}\right.
$$

The objects $\left\{h_{j}\right\},\left\{\lambda_{j}\right\},\left\{y_{j}\right\}$ entering the source term (3.3), are zeros of the nonlinear expression $1+(-1)^{\delta} e^{i Z(\lambda)}$, with the properties as follows:

- holes: $h_{k} \in \mathbb{R}, \quad k=1, \ldots, m_{H}$ are those real solutions of $1+(-1)^{\delta} e^{i Z\left(h_{j}\right)}=0$, which are not Bethe-roots.

- close roots: $c_{k}, \quad k=1, \ldots, m_{C}$, with $0<\left|\operatorname{Im} c_{k}\right| \leq \min (\pi \nu, \pi(1-\nu))$, are complex solutions of $1+(-1)^{\delta} e^{i Z\left(c_{j}\right)}=0$. They are complex zeros of the Bethe-equations.

- wide roots: $w_{k}, \quad k=1, \ldots, m_{W}$, with $\min (\pi \nu, \pi(1-\nu))<\left|\operatorname{Im} w_{k}\right| \leq \frac{\pi}{2}$, are complex Bethe-roots satisfying the equation $1+(-1)^{\delta} e^{i Z\left(w_{j}\right)}=0$.

- special objects: $y_{k} \in \mathbb{R}, k=1, \ldots, m_{S}$ are either holes or real Bethe-roots satisfying the equation $1+(-1)^{\delta} e^{i Z\left(y_{k}\right)}=0$ with $Z^{\prime}\left(y_{k}\right)<0$.

The topological charge $Q$ of the state described by these objects, is given by the so-called counting-equation [39]:

$$
Q=m_{H}-2 m_{S}-m_{C}-2 H(1 / 2-\nu) m_{W},
$$


where here $H(x)$ denotes the Heaviside-function. The equations satisfied by the source objects can be rephrased in their logarithmic form, as well:

$$
\begin{aligned}
& \text { - } \quad \text { holes: } \quad Z\left(h_{k}\right)=2 \pi I_{h_{k}}, \quad I_{h_{k}} \in \mathbb{Z}+\frac{1+\delta}{2}, \quad k=1, \ldots, m_{H} \text {, } \\
& \text { - } \quad \text { close roots: } \quad Z\left(c_{k}\right)=2 \pi I_{c_{k}}, \quad I_{c_{k}} \in \mathbb{Z}+\frac{1+\delta}{2}, \quad k=1, \ldots, m_{C} \text {, } \\
& \text { - } \quad \text { wide roots: } \quad Z\left(w_{k}\right)=2 \pi I_{w_{k}}, \quad I_{w_{k}} \in \mathbb{Z}+\frac{1+\delta}{2}, \quad k=1, \ldots, m_{W}, \\
& \text { - } \quad \text { special objects: } Z\left(y_{k}\right)=2 \pi I_{y_{k}}, \quad I_{y_{k}} \in \mathbb{Z}+\frac{1+\delta}{2}, \quad k=1, \ldots, m_{S} \text {. }
\end{aligned}
$$

This formulation shows, that the actual value of the parameter $\delta \in\{0,1\}$ determines whether the source objects are quantized by integer or half integer quantum numbers. The choice of $\delta$ is not arbitrary, but should follow the selection rules [39]:

$$
\begin{array}{rlrl}
\frac{Q+\delta+M_{\mathrm{sc}}}{2} & \in \mathbb{Z}, & & \text { sine-Gordon, } \\
\frac{\delta+M_{\mathrm{sc}}}{2} & \in \mathbb{Z}, & \text { massive Thirring, }
\end{array}
$$

where here $M_{\mathrm{sc}}$ stands for the number of self-conjugate roots, which are such wide roots, whose imaginary parts are fixed by the periodicity of $Z(\lambda)$ to $\frac{\pi}{2}$. Here we recall, that due to the periodicity of $Z(\lambda)$, the complex roots can be restricted to the domain: $-\frac{\pi}{2}<\lambda_{j} \leq \frac{\pi}{2}$. The form (3.1) is appropriate to impose the quantization equations (3.8), (3.9), (3.11), but to impose the quantization equations (3.10) for the wide roots, the form of (3.1) should be analytically continued to the strip $\min (\pi \nu, \pi(1-\nu))<\operatorname{Im} \lambda \leq \frac{\pi}{2}$, as well. In this domain the equations take the form as follows [36-39]:

$$
\begin{aligned}
& Z(\lambda)=\ell \sinh _{I I} \frac{\lambda}{\nu}+g\left(\lambda \mid\left\{h_{j}\right\},\left\{\lambda_{j}\right\},\left\{y_{j}\right\}\right)_{I I-} \\
& \int_{-\infty}^{\infty} \frac{d \lambda^{\prime}}{i} R_{I I}\left(\lambda-\lambda^{\prime}-i 0 \mid 0\right) L_{+}\left(\lambda^{\prime}+i 0\right)+\int_{-\infty}^{\infty} \frac{d \lambda^{\prime}}{i} R_{I I}\left(\lambda-\lambda^{\prime}+i 0 \mid 0\right) L_{-}\left(\lambda^{\prime}-i 0\right),
\end{aligned}
$$

where II denotes the second determination (3.6).

The energy and momentum of the eigenstates in units of the soliton mass are given by the expressions as follows:

$$
\begin{aligned}
\frac{E(L)-E_{b} \ell}{\mathcal{M}}= & \sum_{j=1}^{m_{H}} \cosh \frac{h_{j}}{\nu}-\sum_{j=1}^{m_{C}} \cosh \frac{c_{j}}{\nu}-\sum_{j=1}^{m_{W}} \cosh I I \frac{w_{j}}{\nu}-\sum_{j=1}^{m_{S}}\left\{\cosh \frac{y_{j}+i 0}{\nu}+\cosh \frac{y_{j}-i 0}{\nu}\right\} \\
& -\int_{-\infty}^{\infty} \frac{d \lambda}{2 \pi \nu i} \sinh \frac{\lambda+i 0}{\nu} L_{+}(\lambda+i 0)+\int_{-\infty}^{\infty} \frac{d \lambda}{2 \pi \nu i} \sinh \frac{\lambda-i 0}{\nu} L_{-}(\lambda-i 0),
\end{aligned}
$$




$$
\begin{aligned}
\frac{P(L)}{\mathcal{M}}= & \sum_{j=1}^{m_{H}} \sinh \frac{h_{j}}{\nu}-\sum_{j=1}^{m_{C}} \sinh \frac{c_{j}}{\nu}-\sum_{j=1}^{m_{W}} \sinh \frac{w_{j}}{\nu}-\sum_{j=1}^{m_{S}}\left\{\sinh \frac{y_{j}+i 0}{\nu}+\sinh \frac{y_{j}-i 0}{\nu}\right\} \\
& -\int_{-\infty}^{\infty} \frac{d \lambda}{2 \pi \nu i} \cosh \frac{\lambda+i 0}{\nu} L_{+}(\lambda+i 0)+\int_{-\infty}^{\infty} \frac{d \lambda}{2 \pi \nu i} \cosh \frac{\lambda-i 0}{\nu} L_{-}(\lambda-i 0),
\end{aligned}
$$

where $L_{ \pm}(\lambda)$ is defined in (3.2) and $E_{b}=\frac{\mathcal{M}}{4} \cot \frac{\pi}{2 \nu}$ is the bulk energy constant.

\section{The function $\omega$ for excited states}

As it will be summarized in the next section, the expectation values of all primaries (2.5) and their Virasoro descendants can be expressed in terms of a single function $\omega(\mu, \lambda \mid \alpha)$ [13]. This function depends on two spectral parameters $\mu, \lambda$, a twist parameter $\alpha$, and although it is not indicated, it depends on the state which sandwiches the operators. Thus it can be considered as a functional of the counting-function of the sandwiching state. The derivation of this function for the ground state can be found in papers $[12,13]$. The result for the sine-Gordon model takes the relatively simple form [13]:

$$
\frac{\omega(\mu, \lambda \mid \alpha)}{4 \pi i}=-\left(F^{+} \star F^{-}\right)(\mu, \lambda)+\left(F^{+} \star R_{d} \star F^{-}\right)(\mu, \lambda),
$$

where

$$
F^{ \pm}(\lambda)=\frac{i}{4 \pi \nu} \frac{1}{\sinh \frac{\lambda \mp i 0}{\nu}},
$$

$R_{d}(\mu, \lambda)$ is the solution of the equation:

$$
R_{d}(\mu, \lambda)+\left(R_{d} \star R\right)(\mu, \lambda)=R(\mu-\lambda \mid \alpha),
$$

where $R(\lambda \mid \alpha)$ is given in (3.4) and the definition of the $\star$ convolution is as follows:

$$
(f \star g)(\mu, \lambda)=\int_{-\infty}^{\infty} d m(t) f(\mu, t) g(t, \lambda),
$$

where the integral measure contains the counting-function:

$$
\begin{aligned}
d m(t) & =\left(\frac{1}{1+a(t-i 0)}+\frac{1}{1+\bar{a}(t+i 0)}\right) d t, \\
a(\lambda) & =(-1)^{\delta} e^{i Z(\lambda)}, \quad \bar{a}(\lambda)=\frac{1}{a(\lambda)}=(-1)^{\delta} e^{-i Z(\lambda)} .
\end{aligned}
$$

Equations (4.1)-(4.5) give the function $\omega$ for the ground state of the model. Now, we extend it to any excited state of the model. To do so, first we introduce the usual convolution as well:

$$
(f * g)(\mu, \lambda)=\int_{-\infty}^{\infty} d t f(\mu, t+i 0) g(t+i 0, \lambda) .
$$


If one starts to analyze the singularity structure of the function $\frac{1}{1+a(\lambda)}$, it turns out, that it has simple poles at the positions of holes or at the positions of the Bethe-roots with residues $\frac{1}{a^{\prime}(\lambda)}$ taken at the position of the singularity. It is known, that the ground state is a state without any hole, such that all Bethe-roots are real. Thus the $\star$ convolution can be written in the following equivalent way:

$$
(f \star g)(\mu, \lambda)=\oint_{\gamma} d t \frac{1}{1+a(t)} f(\mu, t) g(t, \lambda)+(f * g)(\mu, \lambda),
$$

where $\gamma$ is a contour encircling anti-clockwise all the Bethe-roots, but none of the holes. But this formulation of the convolution can be easily extended to excited states, only the contributions of holes and of the complex Bethe-roots should be taken into account by the appropriate application of the residue theorem, when the contours are deformed to run parallel to the real axis. Thus for a generic excited state, the $\star$ convolution takes the form as follows:

$$
(f \star g)(\mu, \lambda)=\int_{-\infty}^{\infty} d m(t) f(\mu, t) g(t, \lambda)-2 \pi i \sum_{j=1}^{m_{H}} \frac{f\left(\mu, h_{j}\right) g\left(h_{j}, \lambda\right)}{a^{\prime}\left(h_{j}\right)}+2 \pi i \sum_{j=1}^{m_{K}} \frac{f\left(\mu, \lambda_{j}\right) g\left(\lambda_{j}, \lambda\right)}{a^{\prime}\left(\lambda_{j}\right)},
$$

where $\lambda_{j}$ denotes the complex Bethe-roots, which are exactly the complex Bethe-roots of the NLIE (3.1): $\left\{\lambda_{j}\right\}_{j=1}^{m_{K}}=\left\{c_{j}\right\}_{j=1}^{m_{C}} \cup\left\{w_{j}\right\}_{j=1}^{m_{W}}$. The only point where the countingfunction, which characterizes the sandwiching state, arises in the formulas for $\omega$, is the convolution. Consequently, the original formulas of (4.1)-(4.3) of [13] remain unchanged for excited states, provided the new definition (4.8) is used for the $\star$ convolution. The only subtle point, which should be clarified is how to interpret the function $R(\mu, \lambda \mid \alpha)$ in (4.3), when its arguments have large enough imaginary parts. To clarify this point, one needs to use another representation of (4.3), which defined $R_{d}(\mu, \lambda)$ for arbitrary complex values of its $\operatorname{arguments}^{2}[12]$;

$$
R_{d}(\mu, \lambda)+\oint_{\gamma} d t \frac{1}{1+a(t)} R_{d}(\mu, t) K_{\alpha}(t-\lambda)=K_{\alpha}(\mu-\lambda), \quad \mu, \lambda \in \mathbb{C},
$$

where here the curve $\gamma$ is the same as in (4.7), the function $K_{\alpha}(\lambda)$ is given by:

$$
K_{\alpha}(\lambda)=\frac{e^{\alpha \lambda}}{2 \pi i}\left(e^{i \pi \nu \alpha}[\operatorname{coth}(\lambda+i \pi \nu)-1]-e^{-i \pi \nu \alpha}[\operatorname{coth}(\lambda-i \pi \nu)-1]\right),
$$

or equivalently in Fourier space:

$$
K_{\alpha}(\lambda)=\int_{-\infty}^{\infty} \frac{d \omega}{2 \pi} e^{i \omega \lambda} \tilde{K}_{\alpha}(\omega), \quad \tilde{K}_{\alpha}(\omega)=\frac{1}{2} \frac{\sinh \left[\frac{\pi \omega}{2}(2 \nu-1)-\frac{i \pi \alpha}{2}\right]}{\sinh \left[\frac{\pi \omega}{2}+\frac{i \pi \alpha}{2}\right]} .
$$

Then using (4.7) and acting on (4.9) with $\left(1-K_{\alpha}\right)^{-1}$ from the right by means of the usual $*$ convolution, one ends up with (4.3). The only subtlety is that one has to take into

\footnotetext{
${ }^{2}$ See $(3.9)$ in $[12]$.
} 
account the poles of $K_{\alpha}(\lambda)$, when the arguments $\mu$ and $\nu$ have large enough imaginary parts. The detailed study of all possible cases shows, that the form of (4.3) remains the valid for arbitrary complex values of $\lambda$ and $\mu$, provided, the kernel $R(\mu, \nu \mid \alpha)$ in (4.3) is defined by the formula:

$$
R(\mu, \lambda \mid \alpha)=K_{\alpha}(\mu-\lambda)+\int_{-\infty}^{\infty} d \tau K_{\alpha}(\mu-\tau) K_{\alpha}(\tau-\lambda)+\int_{-\infty}^{\infty} d \tau d \tau^{\prime} K_{\alpha}(\mu-\tau) R\left(\tau-\tau^{\prime} \mid \alpha\right) K_{\alpha}\left(\tau^{\prime}-\lambda\right),
$$

where on the right hand side $R(\tau \mid \alpha)$ is given by the Fourier integral (3.4). When $\mu$ and $\lambda$ are close to the real axis, from the identity, that $R=K_{\alpha} *\left(1-K_{\alpha}\right)^{-1}$, it can be easily seen, that this definition gives back the original definition (3.4) for $R(\lambda \mid \alpha)$ in the appropriate neighborhood of the real axis.

As it will be clear from the next section, to compute expectation values of local operators, one does not need to have the functional form of $\omega(\mu, \lambda \mid \alpha)$, but only some of the coefficients of its small and/or large argument series representation. Thus, it is worth to define the matrix $\omega_{2 j-1,2 k-1}(\alpha)$ with the definition [13]:

$$
\omega(\mu, \lambda \mid \alpha)=\sum_{j, k=1}^{\infty} e^{-\epsilon_{1} \mu \frac{2 j-1}{\nu}} e^{-\epsilon_{2} \lambda \frac{2 k-1}{\nu}} \omega_{\epsilon_{1}(2 j-1), \epsilon_{2}(2 k-1)}(\alpha), \quad \epsilon_{1,2}= \pm, \quad \epsilon_{1} \mu \rightarrow \infty, \quad \epsilon_{2} \lambda \rightarrow \infty .
$$

Fortunately, to compute the matrix elements $\omega_{2 j-1,2 k-1}$, it is not necessary to solve the equation (4.3) for the two-argument function $R_{d}$. It is enough to solve linear equations for functions of a single variable. Define the function:

$$
e_{j}(\lambda)=\left\{\begin{array}{lr}
e^{j \frac{\lambda}{\nu}}, & |\operatorname{Im} \lambda|<\min [\pi \nu, \pi(1-\nu)], \\
\left(e^{j \frac{\lambda}{\nu}}\right)_{I I}, & \min [\pi \nu, \pi(1-\nu)]<|\operatorname{Im} \lambda|<\frac{\pi}{2} .
\end{array}\right.
$$

Let $\mathcal{G}_{j}(\lambda)$ be the solution of the linear integral equation as follows:

$$
\mathcal{G}_{j}^{(2)}+R \star \mathcal{G}_{j}^{(2)}=e_{j}^{(2)} .
$$

Here for short we introduced an upper index in order to be able to use the short notation (4.8) for the convolution. Thus the interpretation of (4.15) is as follows, each function $\mathcal{G}_{j}, R, e_{j}$ in (4.15) can be considered as two-argument functions, but the upper index "(2)" means that $\mathcal{G}_{j}$ and $e_{j}$ are constant in their second argument, i.e. they are functions of only one variable. Then from (4.1) and (4.3), it can be shown, that the matrix elements of $\omega$ can be expressed in terms of solutions of (4.15) as follows:

$$
\omega_{\epsilon_{1}(2 j-1), \epsilon_{2}(2 k-1)}(\alpha)=\frac{-i}{2 \pi \nu^{2}} e_{\epsilon_{1}(2 j-1)}^{(1)} \star \mathcal{G}_{\epsilon_{2}(2 k-1)}^{(2)}, \quad \epsilon_{1,2}= \pm, \quad j, k \in \mathbb{Z} .
$$

The main advantage of this formula is that, it requires to solve integral equations for functions with a single argument, which is much simpler, than to determine the full function $\omega(\mu, \nu \mid \alpha)$ from (4.1) and (4.3). 


\section{Formulas for the expectation values}

Now, we are in the position to summarize how to compute expectation values of local operators in the fermionic basis in terms of the single function $\omega(\mu, \lambda \mid \alpha)$ defined in the previous section.

Local operators of the theory are labeled by their conformal counterparts. The fermionic basis spans the space of local operators modulo the action of the integrals of motion [13].

In $[12,13]$ it was discovered, that there exist an anti-commuting set of operators acting on the space of local operators of the theory, which allows one to construct an alternative to the Virasoro basis in the Verma-module.

There are two types of fermions for each chirality. The creation and annihilation operators are denoted by $\beta_{j}^{*}, \gamma_{j}^{*}$, and $\beta_{j}, \gamma_{j}$ respectively for one chirality, and by $\bar{\beta}_{j}^{*}, \bar{\gamma}_{j}^{*}$, and $\bar{\beta}_{j}, \bar{\gamma}_{j}$ for the other. The fermions satisfy the anti-commutation relations as follows:

$$
\left\{\beta_{j}, \beta_{k}^{*}\right\}=\left\{\bar{\gamma}_{j}, \bar{\gamma}_{k}^{*}\right\}=\delta_{j k} t_{k}(\alpha), \quad t_{k}(\alpha)=\frac{1}{i \nu} \cot \frac{\pi}{2 \nu}(\nu \alpha+k) .
$$

They span the basis of the Verma module ${ }^{3}$ created by the primary $\Phi_{\alpha+2 m \frac{1-\nu}{\nu}}(0)$, as follows [13]:

$$
\beta_{I^{+}}^{*} \bar{\beta}_{\bar{I}^{+}}^{*} \bar{\gamma}_{\bar{I}^{-}}^{*} \gamma_{I^{-}}^{*} \Phi_{\alpha}^{(m)}(0)
$$

where $\Phi_{\alpha}^{(m)}(0)$ denotes the m-fold screened primary field [47], $I^{ \pm}$and $\bar{I}^{ \pm}$stand for multiindexes, namely:

$$
\begin{aligned}
& I^{+}=\left\{2 i_{1}^{+}-1, \ldots, 2 i_{p}^{+}-1\right\}, \quad \beta_{I^{+}}=\beta_{2 i_{1}^{+}-1}^{*} \ldots \beta_{2 i_{p}^{+}-1}^{*}, \quad 1 \leq i_{1}^{+}<\ldots<i_{p}^{+}, \\
& I^{-}=\left\{2 i_{1}^{-}-1, \ldots, 2 i_{q}^{-}-1\right\}, \quad \gamma_{I^{-}}=\gamma_{2 i_{q}^{-}-1}^{*} \ldots \gamma_{2 i_{1}^{-}-1}^{*}, \quad 1 \leq i_{1}^{-}<\ldots<i_{q}^{-} \text {, }
\end{aligned}
$$

and similarly for the other chirality. For a multi-index $I$, let \#(I) the number of quantum numbers in it. Then in (5.2) the following constraints should hold: $\#\left(I^{+}\right)=\#\left(I^{-}\right)+m$, $\#\left(\bar{I}^{-}\right)=\#\left(\bar{I}^{+}\right)+m$. The lowest dimensional element of the set (5.2) gives the primary field:

$$
\Phi_{\alpha+2 m \frac{1-\nu}{\nu}}(0) \simeq C_{m}(\alpha) \beta_{I_{\mathrm{odd}}(m)}^{*} \bar{\gamma}_{I_{\mathrm{odd}}(m)}^{*} \Phi_{\alpha}^{(m)}(0)
$$

where

$$
I_{\text {odd }}(m)=\{1,3, \ldots, 2 m-1\}
$$

and $C_{m}(\alpha)$ is a constant given in [13] by the formula for $m>0$ :

$$
C_{m}(\alpha)=\prod_{j=0}^{m-1} C_{1}\left(\alpha+2 j \frac{1-\nu}{\nu}\right),
$$

\footnotetext{
${ }^{3}$ More precisely the Verma module factored out by the action of the local integrals of motion [13].
} 
where

$$
\begin{aligned}
& C_{1}(\alpha)=i \nu \Gamma(\nu)^{4 x(\alpha)} \frac{\Gamma(-2 \nu x(\alpha))}{\Gamma(2 \nu x(\alpha))} \frac{\Gamma(x(\alpha))}{\Gamma(x(\alpha)+1 / 2)} \frac{\Gamma(-x(\alpha)+1 / 2)}{\Gamma(-x(\alpha))} \cot (\pi x(\alpha)), \\
& \text { with } \quad x(\alpha)=\frac{\alpha}{2}+\frac{1-\nu}{2 \nu} .
\end{aligned}
$$

For $m<0, C_{m}(\alpha)$ can be determined from the equation [13]:

$$
C_{-m}(\alpha) C_{m}\left(\alpha-2 m \frac{1-\nu}{\nu}\right)=\nu^{2 m} \prod_{j=1}^{m} \tan ^{2}\left(\frac{\pi}{2}\left(\alpha-\frac{j}{\nu}\right)\right) .
$$

The main result of [13] is that the expectation values of the operators (5.2) can be expressed in terms of the matrix-elements (4.16) as follows:

$$
\begin{aligned}
& \frac{\left\langle\beta_{I^{+}}^{*} \bar{\beta}_{\bar{I}^{+}}^{*} \bar{\gamma}_{\bar{I}^{-}}^{*} \gamma_{I^{-}}^{*} \Phi_{\alpha}^{(m)}(0)\right\rangle}{\left\langle\Phi_{\alpha}(0)\right\rangle}= \\
& \mu^{2 m \alpha-2 m^{2}+\frac{1}{\nu}\left(\left|I^{+}\right|+\left|I^{-}\right|+\left|\bar{I}^{+}\right|+\left|\bar{I}^{-}\right|\right)} \mathcal{D}_{R}\left(I^{+} \cup\left(-\bar{I}^{+}\right)\left|I^{-} \cup\left(-\bar{I}^{-}\right)\right| \alpha\right),
\end{aligned}
$$

where $|I|$ denotes the sum of elements of $I$, and for the sets $A=\left\{a_{j}\right\}_{j=1}^{n}, B=\left\{b_{j}\right\}_{j=1}^{n}$, $\mathcal{D}_{R}(A|B| \alpha)$ is given by the determinant:

$$
\begin{aligned}
\mathcal{D}_{R}(A|B| \alpha) & =\operatorname{det} \Omega_{j k}(\alpha), \quad \text { with } \\
\Omega_{j k}(\alpha) & =\omega_{a_{j}, b_{k}}(\alpha)+\frac{i}{\nu} \operatorname{sign}\left(a_{j}\right) \delta_{a_{j}, b_{k}} \cot \left(\frac{\pi}{2 \nu}\left(a_{j}+\nu \alpha\right)\right) .
\end{aligned}
$$

Here $\mu$ is the coupling constant in the action (2.1) of the sine-Gordon model. It is related to the soliton mass $\mathcal{M}$, by the formula [48]:

$$
\mu=\mathcal{M}^{\nu} \Pi(\nu)^{\nu}, \quad \text { where } \quad \Pi(\nu)=\frac{\sqrt{\pi}}{2} \frac{\Gamma\left(\frac{1}{2 \nu}\right)}{\Gamma\left(\frac{1-\nu}{2 \nu}\right)} \Gamma(\nu)^{-\frac{1}{\nu}} .
$$

The expectation values of the descendants of the field $\Phi_{\alpha+2 m \frac{1-\nu}{\nu}}$ can also be computed from (5.9) by the application of the formula [13]:

$$
\begin{aligned}
& \beta_{I^{+}}^{*} \bar{\beta}_{\bar{I}^{+}}^{*} \bar{\gamma}_{\bar{I}^{-}}^{*} \gamma_{I^{-}}^{*} \Phi_{\alpha+2 m \frac{1-\nu}{\nu}}(0) \cong \\
& C_{m}(\alpha) \beta_{I^{+}+2 m}^{*} \bar{\beta}_{\bar{I}^{+}-2 m}^{*} \bar{\gamma}_{\bar{I}^{-}+2 m}^{*} \gamma_{I^{-}-2 m}^{*} \beta_{I_{\text {odd }}(m)}^{*} \bar{\gamma}_{I_{\text {odd }}(m)}^{*} \Phi_{\alpha}^{(m)}(0),
\end{aligned}
$$

where $\cong$ means identification of operators under expectation value and for any "vector" $I$ with elements $\left\{i_{j}\right\}$ the vector $I \pm 2 m$ denotes a vector with elements $\left\{i_{j} \pm 2 m\right\}$. The main formula (5.9) implies, that actually only ratios of expectation values can be computed in terms of the function $\omega$. Nevertheless, as a consequence of (5.4), there is a set of operators whose expectation values can be computed by (5.9). These operators are nothing but the primaries $\Phi_{2 m \frac{1-\nu}{\nu}}$ and their descendants, which form the operator content of the sineGordon model, when it is defined as a perturbed $c=1$ CFT (See section 2.2). Here we just note, that under expectation value, we mean a diagonal matrix element of the operator under consideration, such that the sandwiching eigenstate of the Hamiltonian is normalized to one. 


\section{$6 \quad$ Large volume checks}

In this paper we performed 3 important checks in the large volume limit. Each check is done in the pure multi-soliton sector of the model. The reason for this specialization is that the LeClair-Mussardo type series conjecture of [24] is valid only for the solitonic expectation values of the theory. The 3 checks are as follows. First, we check an identity for the function $\omega(\alpha)$, which ensures the compatibility of the formulas (5.9) and (5.12) in the large volume limit. Then, for the operator $\Phi_{4(1-\nu) / \nu}$ we check up to 3 particle contributions, that the solitonic connected form-factors extracted from the ground state expectation value, are the ones which enter the formulas for the multi-soliton diagonal form-factors as coefficient functions of the different multi-particle densities. Finally, we compute the classical limit of our formula (5.9) for the multi-soliton expectation values of the operators $\Phi_{2 n(1-\nu) / \nu}, n \in \mathbb{N}$, and from the results, we extract the classical limit of connected diagonal form-factors up to two soliton states. The form of these classical connected diagonal form-factors agree with the those coming from direct semi-classical computations in the sine-Gordon model [44].

\subsection{Compatibility check of the formulas (5.9) and (5.12)}

In the original paper [13], it has been shown, that the compatibility of formulas (5.9) and (5.12) require $\omega(\alpha)$ to satisfy the following identities under the analytical continuation of the parameter $\alpha \rightarrow \alpha_{ \pm}=\alpha \pm 2 \frac{1-\nu}{\nu}$ :

$$
\omega_{P Q}\left(\alpha_{ \pm}\right)=\frac{\operatorname{sign}(P \pm 2) \operatorname{sign}(Q \mp 2)}{\operatorname{sign}(P) \operatorname{sign}(Q)}\left[\omega_{P \pm 2, Q \mp 2}(\alpha)-\frac{\omega_{P \pm 2, \mp 1}(\alpha) \omega_{ \pm 1, Q \mp 2}(\alpha)}{\omega_{ \pm 1, \mp 1}(\alpha)+\frac{i}{\nu} \cot \left(\frac{\pi}{2 \nu} \pm \frac{\pi}{2} \alpha\right)}\right] .
$$

This formula is valid for any $P, Q \in \mathbb{Z}$, such that the range of validity in $\alpha$ is restricted to the ranges $0<\alpha<2(1-p)$, and $2 p<\alpha<2$ for the analytical continuations $\alpha \rightarrow \alpha_{+}$and $\alpha \rightarrow \alpha_{-}$, respectively. Here, the parameter $p$ is defined by:

$$
p=\frac{1-\nu}{\nu}, \quad 0<\nu<1
$$

When expectation values in excited states are considered, additional source like terms arise in the equations with respect to the ground state description. The equations with these additional source terms should respect the identities (6.1). Since these source terms are present also in the large volume limit, to show that the excited state equations (4.16) with (4.8) for $\omega(\alpha)$ satisfy (6.1) in the infrared limit, is a nontrivial test on the excited state formulation of the expectation values. In this part, we show that for multi-soliton expectation values, the identities (4.16), are satisfied in the infrared limit.

The first step is to determine $\omega(\alpha)$ in the large volume limit. To do so, equation (4.15) with convolution (4.8) should be solved at large $\ell$. As a consequence of (3.1), in the $\star$ convolution the integral terms become exponentially small in the volume, thus at leading order we neglect them. Thus for large volume and for pure multi-soliton states equation (4.15) 
takes the form:

$$
\mathcal{S}_{s}(\lambda)-2 \pi i \sum_{j=1}^{m} \frac{R\left(\lambda-h_{j} \mid \alpha\right) \mathcal{G}_{s}\left(h_{j}\right)}{a^{\prime}\left(h_{j}\right)}=e_{s}(\lambda) .
$$

It contains the discrete values: $\mathcal{G}_{s}\left(h_{j}\right)$, which can be determined from the linear equations obtained by taking (6.3) at the positions of the holes:

$$
\mathcal{G}_{s}\left(h_{k}\right)-2 \pi i \sum_{j=1}^{m} \frac{R\left(h_{k}-h_{j} \mid \alpha\right) \mathcal{G}_{s}\left(h_{j}\right)}{a^{\prime}\left(h_{j}\right)}=e_{s}\left(h_{k}\right), \quad k=1, \ldots, m .
$$

The solution can be written as:

$$
\mathcal{G}_{s}\left(h_{j}\right)=\sum_{k=1}^{m} a^{\prime}\left(h_{j}\right)\left(\psi^{(\alpha)}(\vec{h})^{-1}\right)_{j k} e_{s}\left(h_{k}\right), \quad j=1, \ldots, m,
$$

where the $\alpha$ dependent $m \times m$ matrix $\psi^{(\alpha)}(\vec{h})$ is of the form:

$$
\psi_{j k}^{(\alpha)}(\vec{h})=a^{\prime}\left(h_{j}\right)-2 \pi i R\left(h_{j}-h_{k} \mid \alpha\right) \quad j, k=1, \ldots, m .
$$

Inserting this into (6.3) one obtains the large volume solution for $\mathcal{G}_{j}(\lambda)$ :

$$
\mathcal{G}_{s}(\lambda)=e_{s}(\lambda)+2 \pi i \sum_{j, k=1}^{m} R\left(\lambda-h_{j} \mid \alpha\right)\left(\psi^{(\alpha)}(\vec{h})^{-1}\right)_{j k} e_{s}\left(h_{k}\right) .
$$

Inserting (6.7) into (4.16), one ends up with the following result for the large volume limit of the matrix $\omega(\alpha)$ :

$$
\omega_{s q}(\alpha)=-\frac{2}{\nu^{2}} \operatorname{sign}(s) \operatorname{sign}(q) \sum_{j, k=1}^{m} e_{s}\left(h_{j}\right)\left(\psi^{(\alpha)}(\vec{h})^{-1}\right)_{j k} e_{q}\left(h_{k}\right)+O\left(e^{-\ell}\right) .
$$

In this limit, the $\alpha$ dependence of $\omega(\alpha)$ is given by the $\alpha$-dependence of the function $R$ in (6.6). We just note, that $a^{\prime}(\lambda)$ is $\alpha$ independent. The key point in the derivation of (6.1), is to know, how $\omega(\alpha)$ changes, when the analytical continuation $\alpha \rightarrow \alpha_{ \pm}=\alpha \pm 2 \frac{1-\nu}{\nu}$ is achieved. Since the $\alpha$ dependence is coming from the function $R$ of (3.4), one should know, how it transforms under the $\alpha \rightarrow \alpha_{ \pm}$analytical continuation.

From (3.4), it is easy to see, that in the Fourier-space the following relations hold:

$$
\tilde{R}\left(\omega \mid \alpha_{ \pm}\right)=\tilde{R}\left(\omega \pm \frac{2 i}{\nu} \mid \alpha\right) .
$$

In the $\lambda$-space, it implies the transformations:

$$
\begin{aligned}
& R\left(\lambda \mid \alpha_{+}\right)=e^{\frac{2 \lambda}{\nu}} R(\lambda \mid \alpha)+\frac{e^{\frac{\lambda}{\nu}}}{\pi \nu} \tan \left[\frac{\pi}{2 \nu}+\frac{\pi \alpha}{\nu}\right]+\sum_{k=1}^{[\alpha / 2+p]} \frac{e^{\left(\frac{2}{\nu}-\frac{2 k-\alpha}{1-\nu}\right) \lambda}}{\pi(1-\nu)} \tan \left[\frac{\pi \nu}{2} \frac{2 k-\alpha}{1-\nu}\right], \\
& R\left(\lambda \mid \alpha_{-}\right)=e^{-\frac{2 \lambda}{\nu}} R(\lambda \mid \alpha)+\frac{e^{-\frac{\lambda}{\nu}}}{\pi \nu} \tan \left[\frac{\pi}{2 \nu}-\frac{\pi \alpha}{\nu}\right]-\sum_{k=[\alpha / 2-p]+1}^{0} \frac{e^{-\left(\frac{2}{\nu}+\frac{2 k-\alpha}{1-\nu}\right) \lambda}}{\pi(1-\nu)} \tan \left[\frac{\pi \nu}{2} \frac{2 k-\alpha}{1-\nu}\right],
\end{aligned}
$$


where [...] stands for integer part in the summation limits. The ranges $0<\alpha<2(1-p)$, and $2 p<\alpha<2$ for the continuations $\alpha \rightarrow \alpha_{+}$and $\alpha \rightarrow \alpha_{-}$, respectively, are chosen to avoid dealing with the last sum in (6.10). Then, the matrix $\psi^{(\alpha)}(\vec{h})$ at the analytically continued points $\alpha_{ \pm}$takes the form:

$$
\psi_{j k}^{\left(\alpha_{ \pm}\right)}(\vec{h})=e_{ \pm 2}\left(h_{j}\right) \tilde{\psi}_{j k}^{\left(\alpha_{ \pm}\right)}(\vec{h}) e_{\mp 2}\left(h_{k}\right), \quad j, k=1, \ldots, m,
$$

where $e_{j}(\lambda)$ is given in (4.14) and

$$
\tilde{\psi}_{j k}^{\left(\alpha_{ \pm}\right)}(\vec{h})=\psi_{j k}^{(\alpha)}(\vec{h})-C_{\nu}^{ \pm} e_{\mp 1}\left(h_{j}\right) e_{ \pm 1}\left(h_{k}\right), \quad \text { with } \quad C_{\nu}^{ \pm}=\frac{2 i}{\nu} \tan \left(\frac{\pi}{2 \nu} \pm \frac{\pi \alpha}{2}\right) .
$$

To express $\omega\left(\alpha_{ \pm}\right)$in terms of $\omega(\alpha)$ from (6.8), the inverse matrix of $\tilde{\psi}_{j k}^{\left(\alpha_{ \pm}\right)}(\vec{h})$ or equivalently of $\psi_{j k}^{\left(\alpha_{ \pm}\right)}(\vec{h})$ should be expressed in terms of the inverse of $\tilde{\psi}_{j k}^{(\alpha)}(\vec{h})$. The determination of the inverse can be done with the help of the following lemma:

Lemma. Let $H$ an $m \times m$ invertible matrix, and $e^{ \pm}$are $m$-dimensional vectors. Then, the inverse of the matrix:

$$
M_{i j}=H_{i j}-C e_{i}^{-} e_{j}^{+}, \quad i, j=1, \ldots, m,
$$

takes the form as follows:

$$
\left(M^{-1}\right)_{i j}=\left(H^{-1}\right)_{i j}-\frac{C}{C e_{r}^{+}\left(H^{-1}\right)_{r q} e_{q}^{-}-1}\left[\left(H^{-1}\right)_{i s} e_{s}^{-}\right]\left[e_{t}^{+}\left(H^{-1}\right)_{t j}\right], \quad i, j=1, \ldots, m,
$$

where for the repeated indexes summation is meant.

The application of the lemma $(6.14)$ to the matrices $\psi^{\left(\alpha_{ \pm}\right)}(\vec{h})$ leads to the following formulas for the inverses:

$$
\left(\psi^{\left(\alpha_{ \pm}\right)}\right)_{j k}^{-1}(\vec{h})=e_{ \pm 2}\left(h_{j}\right)\left(\tilde{\psi}^{\left(\alpha_{ \pm}\right)}\right)_{j k}^{-1}(\vec{h}) e_{\mp 2}\left(h_{k}\right), \quad j, k=1, \ldots, n
$$

where

$$
\left(\tilde{\psi}^{\left(\alpha_{ \pm}\right)}\right)_{j k}^{-1}(\vec{h})=\left(\psi^{(\alpha)}\right)_{j k}^{-1}(\vec{h})-\frac{C_{\nu}^{ \pm} \sum_{l, r=1}^{m}\left[\left(\psi^{(\alpha)}\right)_{j l}^{-1}(\vec{h}) e_{\mp 1}\left(h_{l}\right)\right]\left[e_{ \pm 1}\left(h_{r}\right)\left(\psi^{(\alpha)}\right)_{r k}^{-1}(\vec{h})\right]}{C_{\nu}^{ \pm} \sum_{s, q=1}^{m} e_{ \pm 1}\left(h_{s}\right)\left(\psi^{(\alpha)}\right)_{s q}^{-1}(\vec{h}) e_{\mp 1}\left(h_{q}\right)-1} .
$$

Now, $\omega_{P Q}\left(\alpha_{ \pm}\right)$can be computed by inserting (6.15) into (6.8). The special form of the inverse matrix (6.15) and the identity $e_{j}(h) e_{k}(h)=e_{j+k}(h)$, immediately give the identities (6.1). 


\subsection{Connected diagonal form-factors of $\Phi_{4 \frac{1-\nu}{\nu}}(0)$}

In this subsection, we perform a consistency check on the structure of the large volume expansion of the expectation value formula (5.9). This check is based on the conjecture of [24] for the large volume series representation of expectation values of local operators in pure multi-soliton states. The conjecture was based on the analogous conjecture [19-23] for purely elastic scattering theories. For the sake of completeness we recall it.

Conjecture. Let $\mathcal{O}(x)$ a local operator in the sine-Gordon model. Its expectation value in a pure $n$-soliton state with rapidities $\left\{\theta_{1}, \theta_{2}, \ldots, \theta_{n}\right\}$ can be written as:

$$
\begin{aligned}
\left\langle\theta_{1}, \ldots, \theta_{n}|\mathcal{O}(x)| \theta_{1}, \ldots, \theta_{n}\right\rangle= & \frac{1}{\hat{\rho}\left(\theta_{1}, \ldots, \theta_{n}\right)} \\
& \times \sum_{\left\{\theta_{+}\right\} \cup\left\{\theta_{-}\right\}} \mathcal{D}^{\mathcal{O}}\left(\left\{\theta_{+}\right\}\right) \hat{\rho}\left(\left\{\theta_{-}\right\} \mid\left\{\theta_{+}\right\}\right),
\end{aligned}
$$

where $\hat{\rho}(\vec{\theta})$ is the determinant of the exact Gaudin-matrix:

$$
\hat{\rho}\left(\theta_{1}, \ldots, \theta_{n}\right)=\operatorname{det} \hat{\Phi}(\vec{\theta}), \quad \hat{\Phi}_{k j}(\vec{\theta})=\frac{d}{d \theta_{j}} Z\left(\nu \theta_{k} \mid \nu \vec{\theta}\right), \quad j, k=1, \ldots, m,
$$

$Z(\lambda \mid \vec{h})$ is the counting-function satisfying (3.1) with only hole-type source terms, and the sum in (6.17) runs for all bipartite partitions of the rapidities of the sandwiching state: $\left\{\theta_{1}, \ldots, \theta_{n}\right\}=\left\{\theta_{+}\right\} \cup\left\{\theta_{-}\right\}$, such that

$$
\hat{\rho}\left(\left\{\theta_{+}\right\} \mid\left\{\theta_{-}\right\}\right)=\operatorname{det} \hat{\Phi}_{+}(\vec{\theta}),
$$

with $\hat{\Phi}_{+}(\vec{\theta})$ being the sub-matrix of $\hat{\Phi}(\vec{\theta})$ corresponding to the subset $\left\{\theta_{+}\right\}$. The quantity $\mathcal{D}^{\mathcal{O}}(\{\theta\})$ in (6.17) is called the dressed form-factor [21] and it is given by an infinite sum in terms of the connected diagonal form-factors of the theory:

$$
\begin{aligned}
\mathcal{D}^{\mathcal{O}}\left(\left\{\theta_{1}, \ldots, \theta_{n}\right\}\right) & =\sum_{n_{+}=0}^{\infty} \sum_{n_{-}=0}^{\infty} \frac{1}{n_{+} ! n_{-} !} \int_{-\infty}^{\infty} \prod_{i=1}^{n_{+}+n_{-}} \frac{d \theta_{i}}{2 \pi} \prod_{i=1}^{n_{+}} \mathcal{F}_{+}\left(\theta_{i}+i \eta\right) \prod_{i=n_{+}+1}^{n_{+}+n_{-}} \mathcal{F}_{-}\left(\theta_{i}-i \eta\right) \\
\times & F_{c}^{\mathcal{O}}\left(\theta_{1}, \theta_{2}, \ldots, \theta_{n}, \theta_{1}+i \eta, \ldots, \theta_{n_{+}}+i \eta, \theta_{n_{+}+1}-i \eta, \ldots, \theta_{n_{+}+n_{-}}-i \eta\right)
\end{aligned}
$$

where $F_{c}^{\mathcal{O}}$ denotes the connected diagonal multi-soliton form factors of $\mathcal{O}(x), \eta \in$ $(0, \min (p \pi, \pi))$ is a small contour deformation parameter and $\mathcal{F}_{ \pm}(\theta)$ are the nonlinear expressions of the counting function given by: ${ }^{4}$

$$
\mathcal{F}_{ \pm}(\theta)=\frac{(-1)^{\delta} e^{ \pm i Z(\nu \theta)}}{1+(-1)^{\delta} e^{ \pm i Z(\nu \theta)}}
$$

\footnotetext{
${ }^{4}$ Here the counting-function is used in the $\lambda$ variable and not in the usual rapidity one. The connection is a simple scaling: $\lambda=\nu \theta$.
} 
The idea of the consistency check is as follows. As a consequence of the nonlinear integral equations for $Z(\lambda)$ (3.1), the functions $\mathcal{F}_{ \pm}(\theta \pm i \eta)$ are exponentially small at large volume. Thus the large volume series for a multi-soliton expectation value can be rephrased as an expansion in the functions $\mathcal{F}_{ \pm}(\theta)$. Conjecture (6.20) suggests, that the coefficient functions in this series are the connected diagonal multi-soliton form factors. But, that point of the formula, where a specified connected form-factor enters the series, depend on the state in which the expectation value is taken. This allows us to make a consistency check on (5.9). In papers $[24,25]$ the conjecture was tested for the $\mathrm{U}(1)$ current and for the trace of the stress-energy tensor. Both operators are related to some conserved quantity of the theory. To test the conjecture, we choose a simple operator, which is not related to conserved quantities of the theory. Our choice is the primary: $\Phi_{4(1-\nu) / \nu}(0)$, which is the simplest one in the series $\Phi_{2 m(1-\nu) / \nu}(0)$, after the trace of the stress-energy tensor $T_{\mu}^{\mu} \sim \Phi_{2(1-\nu) / \nu}$. The testing method goes as follows: with the help of (6.17)-(6.20) from the large volume series of the vacuum expectation value, the $k$-particle connected diagonal multi-soliton form-factors can be extracted as coefficient functions of the combination $\mathcal{F}_{+}\left(\theta_{1}\right) \ldots \mathcal{F}_{+}\left(\theta_{k}\right)$. We did it up to 3 -particle contributions. For the first sight, the method seems ambiguous, since everything is valid under integration, but the sought form-factors are symmetric in the rapidities, thus after symmetrization in the rapidities, the results for the connected form-factors become unique.

Having extracted the connected solitonic form-factors from the ground state expectation values, one can check, whether the same functions enter the large volume series expansion of the expectation values in multi-soliton states.

From the ground state expectation value, we determine the first three connected diagonal multi-soliton form-factors of $\Phi_{4(1-\nu) / \nu}(0)$, and check, that the large volume expansion of the expectation value formula (5.9) is consistent with the form of the Bethe-Yang limit of conjecture (6.17)-(6.20) for 1-, 2- and 3-soliton states.

To achieve this computation, one should apply (5.9) for our specific operator $\Phi_{4(1-\nu) / \nu}(0)$, to get a formula for the expectation value. To avoid carrying unnecessary constants, we compute connected solitonic form-factors of the operator:

$$
\bar{\Phi}_{4 \frac{1-\nu}{\nu}}(0)=\frac{\Phi_{4 \frac{1-\nu}{\nu}}(0)}{C_{2}(0) \mu^{\frac{8}{\nu}-8}} .
$$

According to (5.9), its expectation value takes the form:

$$
\left\langle\bar{\Phi}_{4 \frac{1-\nu}{\nu}}(0)\right\rangle=\varphi_{0}+\varphi_{1} \omega_{3,-3}(0)+\varphi_{2} \omega_{1,-1}(0)+\omega_{1,-1}(0) \omega_{3,-3}(0)-\omega_{1,-3}(0) \omega_{3,-1}(0),
$$

where the constants take values:

$$
\varphi_{0}=-\frac{1}{\nu^{2}} \cot \frac{\pi}{2 \nu} \cot \frac{3 \pi}{2 \nu}, \quad \varphi_{1}=\frac{i}{\nu} \cot \frac{\pi}{2 \nu}, \quad \varphi_{2}=\frac{i}{\nu} \cot \frac{3 \pi}{2 \nu} .
$$

This implies, that the large volume expansion of $\left\langle\bar{\Phi}_{4 \frac{1-\nu}{\nu}}(0)\right\rangle$ requires the expansion $\omega_{j k}(0)$ in terms of the functions $\mathcal{F}_{ \pm}(\theta)$. The matrix $\omega_{j k}(\alpha)$ is a functional of the function $\mathcal{G}_{j}(\lambda)$ 
which satisfies (4.15). Thus, to get the large volume series for an expectation value, the following steps should be taken. From (4.15) the large volume series for $\mathcal{G}_{j}(\lambda)$ should be computed. Then one should insert it into the formula (4.16) to get the analogous series for $\omega_{j k}(0)$. Finally, substituting the large volume series representation of $\omega(0)$ into (6.23), gives the required large volume series expression for the expectation value.

Thus, we start the computations by the ground state expectation value, and extract the connected solitonic form-factors from the large volume series representation obtained. The whole computation is very straightforward. The only subtle point is, that one should treat more carefully the integration contour shifts, than it was treated in (4.8) by a $\pm i 0$ prescription in the integration measure. For the ground state $\mathcal{G}_{j}(\lambda)$ satisfies the equation:

$$
\mathcal{G}_{j}(\lambda)=e_{j}(\lambda)-\sum_{\epsilon= \pm} \int_{-\infty}^{\infty} d \tau R(\lambda-\tau-i \epsilon \eta \mid 0) \mathcal{F}_{\epsilon}((\tau+i \epsilon \eta) / \nu) .
$$

To get this equation in the language of $\mathcal{F}_{ \pm}$, we used (6.21) and (4.5). At large volume, the equations can be solved by a straightforward iterative method. The iteration process can be done in the easiest way, by introducing an abstract multi-index notation as follows. For a pair of variables $(\epsilon, \lambda)$, we introduce an abstract capital letter index $A$, such that summation for $A$ unifies summation for $\epsilon$ and integration for $\lambda$ :

$$
(\epsilon, \lambda) \rightarrow A, \quad \sum_{\epsilon= \pm}^{\infty} \int_{-\infty}^{\infty} \rightarrow \sum_{A}
$$

Then it is worth to introduce the "vectors" and "matrices" as follows:

$$
\begin{array}{rlrl}
e_{j}^{(\epsilon)}(\lambda)=e_{j}(\lambda+i \epsilon \eta) & \rightarrow e_{j}^{(A)}, & \\
R_{\epsilon_{1} \epsilon_{2}}\left(\lambda_{1}-\lambda_{2}\right)=R\left(\lambda_{1}-\lambda_{2}+i\left(\epsilon_{1}-\epsilon_{2}\right) \eta \mid 0\right) & \rightarrow R_{A_{1} A_{2},} & \\
\hat{\mathcal{F}}_{\epsilon}(\lambda)=\mathcal{F}_{\epsilon}((\lambda+i \epsilon \eta) / \nu) & \rightarrow \mathcal{F}_{A}, & \\
\mathcal{G}_{j}^{(\epsilon)}(\lambda)=\mathcal{G}_{j}(\lambda+i \epsilon \eta) & \rightarrow \mathcal{G}_{j}^{(A)}, & \\
Q_{A B}=R_{A B} \mathcal{F}_{B}, & & \text { (No summation for B), } \\
\check{e}_{k}^{(A)} & =e_{k}^{(A)} \mathcal{F}_{A} . & & \text { (No summation for A). }
\end{array}
$$

In this language the large volume series for $\mathcal{G}_{j}$ and $\omega(0)$ take the form:

$$
\begin{aligned}
\mathcal{G}_{j}^{(A)} & =(1+Q)_{A B}^{-1} e_{j}^{(B)}, \\
\omega_{j k}(0) & =-\frac{i}{\pi \nu^{2}} \operatorname{sign}(j) \operatorname{sign}(k) \check{e}_{j}^{(A)}(1+Q)_{A C}^{-1} e_{k}^{(C)},
\end{aligned}
$$

where summation is meant for repeated indexes. The matrix $Q=O(\mathcal{F})$, thus the power series of $(1+Q)^{-1}$ admits, the required large volume series:

$$
\omega_{j k}(0)=-\frac{i}{\pi \nu^{2}} \operatorname{sign}(j) \operatorname{sign}(k) \check{e}_{j}^{(A)} \sum_{n=0}^{\infty}(-1)^{n}\left(Q^{n}\right)_{A C} e_{k}^{(C)} .
$$


Inserting (6.29) into (6.23) and returning to the original integration variables, up to $O\left(\mathcal{F}^{3}\right)$ one ends up with the following result for the ground state expectation value:

$$
\begin{aligned}
& \left\langle\bar{\Phi}_{4 \frac{1-\nu}{\nu}}(0)\right\rangle_{0}=\sum_{\epsilon_{1}= \pm} \int_{-\infty}^{\infty} \frac{d \tau}{2 \pi} \hat{\mathcal{F}}_{\epsilon_{1}}(\tau) \bar{F}_{c}^{(1)}\left(\tau^{\left(\epsilon_{1}\right)}\right)+\sum_{\epsilon_{1}, \epsilon_{2}= \pm} \int_{-\infty}^{\infty} \frac{d \tau d \tau^{\prime}}{(2 \pi)^{2}} \hat{\mathcal{F}}_{\epsilon_{1}}(\tau) \hat{\mathcal{F}}_{\epsilon_{2}}\left(\tau^{\prime}\right) \frac{\bar{F}_{c}^{(2)}\left(\tau^{\left(\epsilon_{1}\right)}, \tau^{\prime\left(\epsilon_{2}\right)}\right)}{n_{\epsilon_{1}, \epsilon_{2}}} \\
& +\sum_{\epsilon_{1}, \epsilon_{2}, \epsilon_{3}= \pm} \int_{-\infty}^{\infty} \frac{d \tau d \tau^{\prime} d \tau^{\prime \prime}}{(2 \pi)^{3}} \hat{\mathcal{F}}_{\epsilon_{1}}(\tau) \hat{\mathcal{F}}_{\epsilon_{2}}\left(\tau^{\prime}\right) \hat{\mathcal{F}}_{\epsilon_{3}}\left(\tau^{\prime \prime}\right) \frac{\bar{F}_{c}^{(3)}\left(\tau^{\left(\epsilon_{1}\right)}, \tau^{\prime\left(\epsilon_{2}\right)}, \tau^{\prime \prime\left(\epsilon_{3}\right)}\right)}{n_{\epsilon_{1}, \epsilon_{2}, \epsilon_{3}}}+O\left(\mathcal{F}^{4}\right),
\end{aligned}
$$

where we introduced the short notation $\tau^{(\epsilon)}=\tau+i \epsilon \eta$. The symmetry factors are given as a product of two factorials: $n_{\epsilon_{1}, \ldots}=(\#$ of + indexes $)$ ! (\# of - indexes $)$ !. In the " $\lambda$ convention", the connected diagonal form-factors of $\Phi_{4(1-\nu) / \nu}(0)$ take the form:

$$
\begin{aligned}
\bar{F}_{c}^{(1)}(\tau)= & \frac{2 i}{\nu^{2}}\left(\varphi_{1}+\varphi_{2}\right), \\
\bar{F}_{c}^{(2)}\left(\tau, \tau^{\prime}\right)= & -\frac{4 \pi i}{\nu^{2}} \varphi_{1}\left(e_{3}(\tau) e_{-3}\left(\tau^{\prime}\right)+e_{-3}(\tau) e_{3}\left(\tau^{\prime}\right)\right) R\left(\tau-\tau^{\prime} \mid 0\right) \\
& -\frac{4 \pi i}{\nu^{2}} \varphi_{2}\left(e_{1}(\tau) e_{-1}\left(\tau^{\prime}\right)+e_{-1}(\tau) e_{1}\left(\tau^{\prime}\right)\right) R\left(\tau-\tau^{\prime} \mid 0\right)+\frac{4}{\nu^{4}}\left(e_{2}(\tau) e_{-2}\left(\tau^{\prime}\right)+e_{-2}(\tau) e_{2}\left(\tau^{\prime}\right)-2\right), \\
\bar{F}_{c}^{(3)}\left(\tau_{1}, \tau_{2}, \tau_{3}\right)= & (2 \pi)^{3} \sum_{\sigma \in S_{3}}\left\{\frac{i}{\pi \nu^{2}} \varphi_{1} e_{3}\left(\tau_{\sigma(1)}\right) R_{\sigma(1), \sigma(2)} R_{\sigma(2), \sigma(3)} e_{-3}\left(\tau_{\sigma(3)}\right)\right. \\
& +\frac{i}{\pi \nu^{2}} \varphi_{2} e_{1}\left(\tau_{\sigma(1)}\right) R_{\sigma(1), \sigma(2)} R_{\sigma(2), \sigma(3)} e_{-1}\left(\tau_{\sigma(3)}\right)+\frac{1}{\pi^{2} \nu^{4}}\left[e_{1}\left(\tau_{\sigma(1)}\right) R_{\sigma(1), \sigma(2)} e_{-1}\left(\tau_{\sigma(2)}\right)\right. \\
& +e_{3}\left(\tau_{\sigma(2)}\right) R_{\sigma(2), \sigma(3)} e_{-3}\left(\tau_{\sigma(3)}\right)-e_{1}\left(\tau_{\sigma(1)}\right) R_{\sigma(1), \sigma(2)} e_{-3}\left(\tau_{\sigma(2)}\right) e_{2}\left(\tau_{\sigma(3)}\right) \\
& \left.\left.-e_{-2}\left(\tau_{\sigma(1)}\right) e_{3}\left(\tau_{\sigma(2)}\right) R_{\sigma(2), \sigma(3)} e_{-1}\left(\tau_{\sigma(3)}\right)\right]\right\}
\end{aligned}
$$

where in the expression of $\bar{F}_{c}^{(3)}$, the sum runs for all permutations of the 3 indexes, and we introduced the short notation $R_{j k}=R\left(\tau_{j}-\tau_{k} \mid 0\right)$.

Now, one can compute the large volume limit of the expectation values from (5.9) for multi-soliton states. From (6.7) and (6.8), for $\omega(0)$, the result as follows is obtained:

$$
\omega_{j k}^{B Y}(0)=-\frac{2 i}{\nu^{2}} \operatorname{sign}(j) \operatorname{sign}(k) \sum_{s, q=1}^{m} e_{j}\left(h_{s}\right) \Phi_{s q}^{-1}(h) e_{k}\left(h_{q}\right),
$$

where $e_{j}(h)$ is defined in (4.14) and the Gaudin-matrix is given by:

$$
\begin{array}{cc}
\Phi_{j k}(h)=\left\{\begin{aligned}
\rho_{1}\left(h_{j}\right)-2 \pi \sum_{s \neq j}^{m} R\left(h_{j}-h_{s} \mid 0\right), & j=k, \\
2 \pi R\left(h_{j}-h_{k} \mid 0\right), & j \neq k,
\end{aligned}\right. \\
\text { with } \\
\rho_{1}(h)=\frac{\ell}{\nu} \cosh \frac{h}{\nu} .
\end{array}
$$


The positions of the holes in this formula are related to the rapidity variables of (6.17) by $\frac{h_{j}}{\nu}=\theta_{j}$. We note, that here $\Phi_{j k}$ is the Gaudin-matrix in the $\lambda$ variable, which is related to that of in rapidity variable by a scaling with $\nu: \Phi_{j k}\left(\nu \theta_{1}, \ldots\right)=\frac{1}{\nu} \hat{\Phi}_{j k}\left(\theta_{1}, \ldots\right)$. As a consequence, the corresponding $n$-particle densities are related by a scaling with $\nu^{n}: \rho^{(n)}\left(\nu \theta_{1}, \nu \theta_{1}, \ldots\right)=\frac{1}{\nu^{n}} \hat{\rho}^{(n)}\left(\theta_{1}, \theta_{2}, \ldots\right)$.

From inserting (6.33) into (6.23), the large volume limit of the expectation values of $\bar{\Phi}_{4 \frac{1-\nu}{\nu}}(0)$, in 1-, 2-, and 3-soliton states can be determined. For 1-particle the result takes the form:

$$
\left\langle h_{1}\left|\bar{\Phi}_{4 \frac{1-\nu}{\nu}}(0)\right| h_{1}\right\rangle=\varphi_{0}+\frac{1}{\operatorname{det} \Phi\left(h_{1}\right)} \bar{F}_{c}^{(1)}\left(h_{1}\right) .
$$

For the 2-particle expectation value, one obtains:

$$
\left\langle h_{1}, h_{2}\left|\bar{\Phi}_{4 \frac{1-\nu}{\nu}}(0)\right| h_{1}, h_{2}\right\rangle=\varphi_{0}+\frac{\bar{F}_{c}^{(1)}\left(h_{1}\right) \rho^{(1)}\left(h_{2}\right)+\bar{F}_{c}^{(1)}\left(h_{2}\right) \rho^{(1)}\left(h_{1}\right)+\bar{F}_{c}^{(2)}\left(h_{1}, h_{2}\right)}{\operatorname{det} \Phi\left(h_{1}, h_{2}\right)},
$$

where $\rho^{(1)}\left(h_{j}\right)=\frac{\ell}{\nu} \cosh \frac{h_{j}}{\nu}-2 \pi R\left(h_{1}-h_{2} \mid 0\right), j=1,2$.

For the 3-particle case the result is as follows:

$$
\begin{gathered}
\left\langle h_{1}, h_{2}, h_{3}\left|\bar{\Phi}_{4 \frac{1-\nu}{\nu}}(0)\right| h_{1}, h_{2}, h_{3}\right\rangle=\varphi_{0}+\frac{1}{\operatorname{det} \Phi\left(h_{1}, h_{2}, h_{3}\right)}\left\{\bar{F}_{c}^{(1)}\left(h_{1}\right) \rho_{3}^{(2)}\left(h_{2}, h_{3} \mid h_{1}\right)\right. \\
+\bar{F}_{c}^{(1)}\left(h_{2}\right) \rho_{3}^{(2)}\left(h_{1}, h_{3} \mid h_{2}\right)+\bar{F}_{c}^{(1)}\left(h_{3}\right) \rho_{3}^{(2)}\left(h_{1}, h_{2} \mid h_{3}\right)+\bar{F}_{c}^{(2)}\left(h_{1}, h_{2}\right) \rho_{3}^{(1)}\left(h_{3} \mid h_{1}, h_{2}\right) \\
\left.+\bar{F}_{c}^{(2)}\left(h_{1}, h_{3}\right) \rho_{3}^{(1)}\left(h_{2} \mid h_{1}, h_{3}\right)+\bar{F}_{c}^{(2)}\left(h_{2}, h_{3}\right) \rho_{3}^{(1)}\left(h_{1} \mid h_{2}, h_{3}\right)+\bar{F}_{c}^{(3)}\left(h_{1}, h_{2}, h_{3}\right)\right\},
\end{gathered}
$$

where the densities take the forms:

$$
\begin{aligned}
& \rho_{3}^{(1)}\left(h_{j} \mid h_{k}, h_{l}\right)=\rho_{j}-\hat{R}_{j k}-\hat{R}_{j l}, \\
& \rho_{3}^{(2)}\left(h_{k}, h_{l} \mid h_{j}\right)=\rho_{k} \rho_{l}-\rho_{k}\left(\hat{R}_{k l}+\hat{R}_{l j}\right)-\rho_{l}\left(\hat{R}_{k l}+\hat{R}_{k j}\right)+\hat{R}_{k l} \hat{R}_{l j}+\hat{R}_{k l} \hat{R}_{k j}+\hat{R}_{k j} \hat{R}_{l j}, \\
& \text { with } \quad \rho_{j}=\frac{\ell}{\nu} \cosh \frac{h_{j}}{\nu}, \quad \text { and } \quad \hat{R}_{j k}=2 \pi R\left(h_{j}-h_{k} \mid 0\right) .
\end{aligned}
$$

The results (6.35)-(6.37) are in perfect agreement with the conjecture (6.17).

To summarize the results, we close this section by listing the multi-soliton connected diagonal form-factors of the operator $\Phi_{4 \frac{1-\nu}{\nu}}(0)$ in rapidity variables. They take the form as follows:

$$
\begin{aligned}
F_{c}^{(0)}(\emptyset) & =C_{2}(0) \mu^{\frac{8}{\nu}-8} \varphi_{0}, \\
F_{c}^{(1)}\left(\theta_{1}\right) & =C_{2}(0) \mu^{\frac{8}{\nu}-8} \nu \bar{F}_{c}^{(1)}\left(\nu \theta_{1}\right), \\
F_{c}^{(2)}\left(\theta_{1}, \theta_{2}\right) & =C_{2}(0) \mu^{\frac{8}{\nu}-8} \nu^{2} \bar{F}_{c}^{(2)}\left(\nu \theta_{1}, \nu \theta_{2}\right), \\
F_{c}^{(3)}\left(\theta_{1}, \theta_{2}, \theta_{3}\right) & =C_{2}(0) \mu^{\frac{8}{\nu}-8} \nu^{3} \bar{F}_{c}^{(3)}\left(\nu \theta_{1}, \nu \theta_{2}, \nu \theta_{3}\right),
\end{aligned}
$$

with the functions $\bar{F}_{c}\left(h_{1}, \ldots\right)$ given in (6.31) and (6.32). Here the prefactor in front of the functions $\bar{F}_{c}$ comes from either the proper normalization factor of the operator $\Phi_{4 \frac{1-\nu}{\nu}}(0)$ and the transformation from the $\lambda$-type variables to the rapidity variables. 


\subsection{Classical limit of connected diagonal form factors}

In this subsection, starting from the formulas (5.4) and (5.9), we compute the classical limit of the multi-soliton connected diagonal form-factors of the operators $\Phi_{2 n \frac{1-\nu}{\nu}}(0)$ and compare the results with the direct semi-classical computation of reference [44].

The connected diagonal form-factors can be extracted from the expectation values with the help of the Bethe-Yang limit of the conjectured expectation value formula (6.17)-(6.21).

For pure soliton states, in the Bethe-Yang limit the expectation values take exactly the same form as those in a purely elastic scattering theory $[8,17,22]$. This is in accordance with the earlier conjecture of [18], for the Bethe-Yang limit of expectation values in nondiagonally scattering theories.

For an $m$-soliton state with rapidities $\left\{\theta_{1}, \theta_{2}, \ldots, \theta_{m}\right\}$ the large volume limit of an expectation value takes the form:

$$
\begin{aligned}
\left\langle\theta_{1}, \ldots, \theta_{m}|\mathcal{O}(x)| \theta_{1}, \ldots, \theta_{m}\right\rangle= & \frac{1}{\rho\left(\theta_{1}, \ldots, \theta_{m}\right)} \\
& \times \sum_{\left\{\theta_{+}\right\} \cup\left\{\theta_{-}\right\}} F_{c}^{\mathcal{O}}\left(\left\{\theta_{+}\right\}\right) \rho\left(\left\{\theta_{-}\right\} \mid\left\{\theta_{+}\right\}\right),
\end{aligned}
$$

where again the sum in (6.40) runs for all bipartite partitions of the rapidities of the sandwiching state: $\left\{\theta_{1}, \ldots, \theta_{n}\right\}=\left\{\theta_{+}\right\} \cup\left\{\theta_{-}\right\}$, such that the partial densities are made out of the infinite volume Gaudin-matrix of (6.34) by the formula;

$$
\rho\left(\left\{\theta_{+}\right\} \mid\left\{\theta_{-}\right\}\right)=\operatorname{det} \Phi_{+}(\vec{\theta}),
$$

with $\Phi_{+}(\vec{\theta})$ being the sub-matrix of $\Phi(\vec{\theta})$ corresponding to the subset $\left\{\theta_{+}\right\}$. Now, the coefficients of the densities are directly the connected diagonal form-factors $F_{c}^{\mathcal{O}}(\{\theta\})$.

The strategy of computing the classical limit of the connected diagonal form-factors up to two soliton-states is as follows. From (5.4) and (5.9), one can write down explicit formulas for the expectation values of $\mathcal{O}_{n}=\Phi_{2 n \frac{1-\nu}{\nu}}$ in multi-soliton states. First, one should take the vacuum expectation value. Then from (6.40) at $m=0$, one obtains $F_{0, c}^{\mathcal{O}_{n}}(\{\emptyset\})$. Then by comparing the 1-particle expectation value and (6.40) applied for $m=1$, and by knowing $F_{0, c}^{\mathcal{O}_{n}}(\{\emptyset\})$, one can extract the 1-particle connected form-factors: $F_{1, c}^{\mathcal{O}_{n}}(\{\theta\})$. As a last step, the 2-particle expectation value should be compared to (6.40) at $m=2$, and by knowing $F_{0, c}^{\mathcal{O}_{n}}(\{\emptyset\})$, and $F_{1, c}^{\mathcal{O}_{n}}(\{\theta\})$, one can extract the 2-particle connected form-factors: $F_{2, c}^{\mathcal{O}_{n}}\left(\left\{\theta_{1}, \theta_{2}\right\}\right)$. Finally, the classical limit should be taken in the resulting connected formfactors.

For general values of $n$, and for more than 2 soliton states, the computation described above would be very complicated to achieve. Nevertheless, as we will see in the case of two-soliton expectation values, the whole computation can be simplified, if the classical limit is taken appropriately in the internal steps of the computation.

To treat the classical limit appropriately, as a first step, we collect some important formulas concerning the classical limit of some basic building blocks of the computations. The classical limit is the $\nu \rightarrow 1$ or equivalently the $p \rightarrow 0$ limit of the theory. In this limit 
both the normalization factor $C_{n}(\alpha)$ in (5.6) and the kernel $R(\lambda \mid 0)$ given in (3.4) diverge:

$$
\begin{aligned}
C_{n}(0) & =\frac{1}{(\pi p)^{n}} C_{n}^{\text {class }}(0)+\ldots, \quad \text { with } \\
C_{n}^{\text {class }}(0) & =\frac{(2 i)^{n}}{\prod_{j=1}^{n}(2 j-1)}, \\
2 \pi R(\lambda \mid 0) & =\frac{-1}{\pi p}\left(2 \ln \tanh \frac{\lambda}{2}+\ldots\right) .
\end{aligned}
$$

The expectation value of the operators $\mathcal{O}_{n}(0)$ requires the matrix $\omega_{j k}(0)$ of $(4.13)$ in the $\ell \rightarrow \infty$ limit. This is given in formula (6.33).

The application of the formulas (5.4) and (5.9) gives the following representation for the Bethe-Yang limit of expectation values of the operators $\mathcal{O}_{n}=\Phi_{2 n \frac{1-\nu}{\nu}}$ :

$$
\left\langle O_{n}(0)\right\rangle=C_{n}(0) \operatorname{det}_{n \times n} M^{\text {(quant) }},
$$

where $M^{\text {(quant) }}$ denotes the Bethe-Yang limit of $\Omega_{j k}$ in (5.10):

$$
M_{j k}^{\text {(quant) }}=\omega_{2 j-1,1-2 k}^{B Y}+\frac{i}{\nu} \cot \left[\frac{\pi}{2 \nu}(2 j-1)\right] \delta_{j k}, \quad j, k=1, \ldots, n .
$$

Using this formula, the vacuum expectation value can be computed with ease. In this case there are no particles present, thus $\omega^{B Y}=0$, allowing to compute the necessary determinant easily:

$$
\left\langle\mathcal{O}_{n}(0)\right\rangle_{0}=C_{n}(0)\left(\frac{i}{\nu}\right)^{n} \prod_{j=1}^{n} \cot \left[\frac{\pi}{2 \nu}(2 j-1)\right] \stackrel{p \rightarrow 0}{=} 1+O(\pi p) .
$$

Comparing (6.45) and (6.40) gives the classical limit of the 0-particle connected formfactors:

$$
F_{c, \text { class }}^{\mathcal{O}_{n}}(\{\emptyset\})=1
$$

The computation of the 1-soliton connected form-factor goes as follows. Let $A$ a diagonal matrix:

$$
A_{j k}=A_{j} \delta_{j k}, \quad A_{j}=\frac{i}{\nu} \cot \left[\frac{\pi}{2 \nu}(2 j-1)\right], \quad j, k=1, \ldots, n .
$$

In this case the matrix $\omega_{2 j-1,1-2 k}^{B Y}$ takes the form of a dyadic product:

$$
\begin{aligned}
\omega_{2 j-1,1-2 k}^{B Y} & =\frac{2 i}{\nu^{2}} \frac{1}{\rho_{1}(h)} e_{j}^{+}(h) e_{k}^{-}(h), & j, k & =1, \ldots, n, \\
e_{j}^{ \pm}(h) & =e^{ \pm \frac{2 j}{\nu} h}, & j & =1, \ldots, n,
\end{aligned}
$$

where $\rho_{1}(h)$ is given in (6.34). The matrix $M^{\text {(quant) }}$ takes the special form:

$$
M^{\text {(quant) }}=A+\lambda_{0} e^{+} \circ e^{-}, \quad \text { with } \quad \lambda_{0}=\frac{2 i}{\nu^{2}} \frac{1}{\rho_{1}(h)},
$$


where $\odot$ stands for the dyadic product. The determinant of such a matrix is given by the simple formula:

$$
\operatorname{det}\left(A+\lambda_{0} e^{+} \circ e^{-}\right)=\operatorname{det} A \cdot\left(1+\lambda_{0} e^{+} A^{-1} e^{-}\right) .
$$

With the help of this formula, one obtains the result as follows for the 1-soliton expectation value,

$$
\left\langle\mathcal{O}_{n}(0)\right\rangle_{1}=F_{0, c}^{\mathcal{O}_{n}}(\emptyset)+\frac{F_{0, c}^{\mathcal{O}_{n}}(\emptyset)}{\rho_{1}(h)}\left(\frac{2 i}{\nu^{2}} \sum_{j=1}^{n} \frac{1}{A_{j}}\right)
$$

where we exploited the previous result (6.45) for the vacuum expectation value: $F_{0, c}^{\mathcal{O}_{n}}(\emptyset)=$ $C_{n}(0) \operatorname{det} A$. Comparing (6.51) to (6.40) applied for $m=1$, the 1-particle connected diagonal form-factor can be obtained:

$$
F_{1, c}^{\mathcal{O}_{n}}(\theta)=F_{0, c}^{\mathcal{O}_{n}}(\emptyset)\left(\frac{2 i}{\nu^{2}} \sum_{j=1}^{n} \frac{1}{A_{j}}\right) \stackrel{p \rightarrow 0}{=}-\frac{1}{\pi p} F_{c, \text { class }}^{\mathcal{O}_{n},(0)}(\emptyset) 4 \sum_{j=1}^{n} \frac{1}{2 j-1} .
$$

This result agrees with that of the classical computations [44] for the 1-particle solitonic connected diagonal form-factors. From (6.52), it can bee seen that the 1-particle connected form-factors of $\mathcal{O}_{n}(0)$ scale as $\frac{1}{\pi p}$ in the classical limit.

As the number of sandwiching solitons increase, it is helpful for the computations to know, how the connected form-factors scale in the classical limit. As a consequence of the scaling (6.42) of the function $R(\lambda \mid 0)$, the multi-soliton connected form-factors admit the following scaling property in the classical limit: ${ }^{5}$

$$
F_{m, c}^{\mathcal{O}_{n}}\left(\theta_{1}, \ldots, \theta_{m}\right) \stackrel{p \rightarrow 0}{=} \frac{1}{(\pi p)^{m}} F_{c, \text { class }}^{\mathcal{O}_{n},(m)}\left(\theta_{1}, \ldots, \theta_{m}\right)+O\left((\pi p)^{1-m}\right)
$$

where $F_{c, \text { class }}^{\mathcal{O}_{n},(m)}\left(\theta_{1}, \ldots, \theta_{m}\right)$ is what we call now the classical limit of connected form factors. Beyond the level of 1-particle expectation values, the computations become so complicated, that to simplify them, it is worth to exploit this scaling property at the internal steps of the computations.

For the 2-soliton expectation values, as a first step we write down the densities appearing in (6.40). We consider (6.40) as the function of particle's rapidities and the corresponding 1-particle densities. Thus, we write down the Bethe-Yang limit of the 2-particle Gaudin-matrix in this spirit:

$$
\hat{\Phi}\left(\theta_{1}, \theta_{2}\right)=\left(\begin{array}{cc}
\rho_{1}\left(\nu \theta_{1}\right)-2 \pi \nu R\left(\nu\left(\theta_{1}-\theta_{2}\right) \mid 0\right) & 2 \pi \nu R\left(\nu\left(\theta_{1}-\theta_{2}\right) \mid 0\right) \\
2 \pi \nu R\left(\nu\left(\theta_{1}-\theta_{2}\right) \mid 0\right) & \rho_{1}\left(\nu \theta_{2}\right)-2 \pi \nu R\left(\nu\left(\theta_{1}-\theta_{2}\right) \mid 0\right)
\end{array}\right) .
$$

\footnotetext{
${ }^{5}$ This scaling property can be seen in two ways. First, for fixed low values of $n$, one can do the computations such that the classical limit is taken only at the end. And for each case this scaling is obtained. (E.g. for $n=2$, the quantum result can be found in the previous subsection.) Second, the solitonic connected diagonal form-factors of $\mathcal{O}_{1}(0)$ are known explicitly, since this operator is proportional to the trace of the stress-energy tensor. They are composed of simple products of the function $R(\lambda \mid 0)$, which allows one to compute easily the classical limit.
} 
From (6.42) and (6.34), one can see, that $\rho_{1}(\theta) \sim O(1)$, while $R(\nu \theta \mid 0) \sim O\left(\frac{1}{\pi p}\right)$ in the classical limit. Thus for computational reasons it is worth to rewrite $\hat{\Phi}\left(\theta_{1}, \theta_{2}\right)$ in terms of a scaled 1-particle density:

$$
\rho_{c}(\theta)=\pi p \rho_{1}(\nu \theta)
$$

One can write down (6.40) in terms of the rapidities and the new densities $\rho_{c}(\theta)$ and take the $p \rightarrow 0$ classical limit such that $\rho_{c}(\theta)$ and the rapidities are kept finite. In the classical limit the Gaudin-matrix will behave as follows:

$$
\begin{aligned}
\hat{\Phi}\left(\theta_{1}, \theta_{2}\right) & \stackrel{p \rightarrow 0}{=} \frac{1}{\pi p} \hat{\Phi}^{\mathrm{cl}}\left(\theta_{1}, \theta_{2}\right)+\ldots, \\
\hat{\Phi}^{\mathrm{cl}}\left(\theta_{1}, \theta_{2}\right) & =\left(\begin{array}{cc}
\rho_{c}\left(\theta_{1}\right)+2 \ln u & -2 \ln u \\
-2 \ln u & \rho_{c}\left(\theta_{2}\right)+2 \ln u
\end{array}\right), \quad u=\tanh \frac{\theta_{1}-\theta_{2}}{2} .
\end{aligned}
$$

Then the densities entering (6.40) take the form:

$$
\begin{gathered}
\rho_{2}\left(\theta_{1}, \theta_{2}\right)=\frac{1}{(\pi p)^{2}} \operatorname{det} \hat{\Phi}^{\mathrm{cl}}\left(\theta_{1}, \theta_{1}\right)+\ldots, \\
\rho\left(\theta_{1} \mid \theta_{2}\right)=\frac{1}{\pi p}\left(\rho_{c}\left(\theta_{1}\right)+2 \ln u\right)+\ldots, \\
\rho\left(\theta_{2} \mid \theta_{1}\right)=\frac{1}{\pi p}\left(\rho_{c}\left(\theta_{2}\right)+2 \ln u\right)+\ldots
\end{gathered}
$$

Inserting (6.57) and the scaling property (6.53) into (6.40), the expectation value can be written in the $p \rightarrow 0$ limit as follows:

$$
\begin{gathered}
\left\langle\mathcal{O}_{n}(0)\right\rangle_{2} \stackrel{p \rightarrow 0}{=} F_{c, \text { class }}^{\mathcal{O}_{n},(0)}(\emptyset)+\frac{1}{\operatorname{det} \hat{\Phi}^{\mathrm{cl}}\left(\theta_{1}, \theta_{2}\right)}\left(F_{c, \text { class }}^{\mathcal{O}_{n},(2)}\left(\theta_{1}, \theta_{2}\right)+F_{c, \text { class }}^{\mathcal{O}_{n},(1)}\left(\theta_{1}\right)\left(\rho_{c}\left(\theta_{1}\right)+2 \ln u\right)+\right. \\
\left.F_{c, \text { class }}^{\mathcal{O}_{n},(1)}\left(\theta_{2}\right)\left(\rho_{c}\left(\theta_{2}\right)+2 \ln u\right)\right)+\ldots
\end{gathered}
$$

With the help of (6.58) the extraction of the classical 2-soliton connected form-factor goes as follows. The expectation value $\left\langle\mathcal{O}_{n}(0)\right\rangle_{2}$ is considered as a function of many parameters:

$$
\left\langle\mathcal{O}_{n}(0)\right\rangle_{2}=\mathbf{F}\left(\rho_{c, 1}, \rho_{c, 2}, u \mid p\right), \quad \text { where } \quad \rho_{c, j}=\rho_{c}\left(\theta_{j}\right) .
$$

(6.56) suggests a similar reparameterization for the classical 2-particle density:

$$
\operatorname{det} \hat{\Phi}^{\mathrm{cl}}\left(\theta_{1}, \theta_{2}\right)=\rho_{2}\left(\rho_{c, 1}, \rho_{c, 2}, u \mid p\right) .
$$

Formula (6.58) implies, that the classical 2-soliton connected form-factor can be obtained as follows:

$$
F_{c, \text { class }}^{\mathcal{O}_{n},(2)}\left(\theta_{1}, \theta_{2}\right)=\lim _{\rho \rightarrow 0} \mathbf{F}(\rho, \rho, u \mid 0) \rho_{2}(\rho, \rho, u \mid 0)-4 F_{c, \text { class }}^{\mathcal{O}_{n},(1)}\left(\theta_{1}\right) \ln u .
$$


We just note, that we exploited, that the 1-particle connected form-factor is independent of the rapidity. Finally formula (6.60) together with (6.56), (6.43), (6.33), (6.44) admit the following representation for the 2 -soliton diagonal connected form-factors in the variable $u=\tanh \frac{\theta_{1}-\theta_{2}}{2}$ :

$$
F_{c, \text { class }}^{\mathcal{O}_{n},(2)}(u)=C_{n}^{\text {class }}(0) \lim _{\rho \rightarrow 0} \operatorname{det} M^{(\text {class })}(u \mid \rho) \operatorname{det} \Phi^{\mathrm{cl}}(u \mid \rho)-4 F_{c, \text { class }}^{\mathcal{O}_{n},(1)}\left(\theta_{1}\right) \ln u,
$$

where the matrix $\Phi^{\mathrm{cl}}(u \mid \rho)$ is equal to $\hat{\Phi}^{\mathrm{cl}}$ of $(6.56)$ taken at $\rho_{c, 1}=\rho_{c, 2}=\rho$ :

$$
\Phi^{\mathrm{cl}}(u \mid \rho)=\left(\begin{array}{cc}
\rho+2 \ln u & -2 \ln u \\
-2 \ln u & \rho+2 \ln u
\end{array}\right), \quad u=\tanh \frac{\theta_{1}-\theta_{2}}{2},
$$

the matrix $M^{\text {(class) }}(u \mid \rho)$ can be considered as the classical limit of $M^{\text {(quant) }}$ of (6.44). It is expressed i term of the classical limit of $\omega_{j k}^{B Y}$ :

$$
\omega_{j k}^{B Y}(u \mid \rho)=-2 i \operatorname{sign}(j) \operatorname{sign}(k)\left\{t(u)^{j+k} \Phi_{11}^{\mathrm{cl},-1}+\Phi_{22}^{\mathrm{cl},-1}+t(u)^{j} \Phi_{12}^{\mathrm{cl},-1}+t(u)^{k} \Phi_{21}^{\mathrm{cl},-1}\right\},
$$

where $t(u)=\frac{u+1}{u-1}$ and $\Phi_{j k}^{\mathrm{cl},-1}$ denotes the $j k$ matrix element of the inverse of $\Phi^{\mathrm{cl}}(u \mid \rho)$ of $(6.63)$. Then $M^{\text {(class) }}(u \mid \rho)$ is given by the formula:

$$
M^{(\text {class })}(u \mid \rho)=\omega_{2 j-1,1-2 k}^{B Y}(u \mid \rho)-\frac{i}{2}(2 j-1) \delta_{j k}, \quad j, k=1, \ldots, n .
$$

For the first few values of $n$, we just list the classical limit of connected diagonal 2-soliton form factors:

$$
\frac{F_{c, \text { class }}^{\mathcal{O}_{n},(2)}(u)}{F_{c, \text { class }}^{\mathcal{O}_{n},(0)}(\emptyset)}=\left\{\begin{aligned}
\frac{16\left(u^{2}+1\right) \log (u)}{u^{2}-1}, & n=1, \\
-\frac{256 u^{2}}{3\left(u^{2}-1\right)^{2}}+\frac{64\left(u^{2}+1\right)^{3} \log (u)}{3\left(u^{2}-1\right)^{3}}, & n=2, \\
-\frac{512 u^{2}\left(3 u^{4}+2 u^{2}+3\right)}{5\left(u^{2}-1\right)^{4}}+\frac{16\left(u^{2}+1\right)\left(23 u^{8}+132 u^{6}+458 u^{4}+132 u^{2}+23\right) \log (u)}{15\left(u^{2}-1\right)^{5}}, & n=3, \\
& \text { etc... }
\end{aligned}\right.
$$

We compared these results up to $n=8$ with the ones obtained by semi-classical commutations in [44], and found perfect agreement. ${ }^{6}$

\section{Small volume checks}

In this section in the pure multi-soliton sector, we compare our results to 3-point functions of the Liouville theory on the cylinder (see figure 1). Let $\left\{h_{j}\right\}_{j=1}^{n}$ the rapidities of the pure multi-soliton state. We consider the ultraviolet limit of the expectation value

\footnotetext{
${ }^{6}$ The semi-classical results of Bajnok and Janik can be found in [44] up to $n=4$. For higher values of $n$, we could use the unpublished Mathematica notebook file of the authors.
} 
$\frac{\left\langle h_{1}, \ldots, h_{n}|\mathcal{O}(0)| h_{1}, \ldots h_{n}\right\rangle}{\left\langle h_{1}, \ldots, h_{n} \mid h_{1}, \ldots, h_{n}\right\rangle}$. The operator under consideration is a primary field or a Virasoro descendant of a primary field: $\mathcal{O}(0) \rightarrow \mathbf{l}_{-k_{1}} \ldots \mathbf{l}_{-k_{j}} \Phi_{\alpha}(0)$, and the sandwiching state tends to a primary state or a descendant state of the theory: $\left|h_{1}, \ldots h_{n}\right\rangle \rightarrow L_{-n_{1}} \ldots L_{-n_{i}}|\Delta\rangle$. If we introduce the complex coordinate on the cylinder of radius one as $z=x+i y$, such that $y \equiv y+2 \pi$, then in the previous lines $\mathbf{l}_{n}$ and $L_{n}$ are the coefficients of the Laurent-expansion of the stress-energy tensor $T(z)$ around the origin and $z \rightarrow \pm \infty$, respectively:

$$
T(z)=\sum_{n=-\infty}^{\infty} \mathbf{l}_{n} z^{-n-2}, \quad T(z)=\sum_{n=-\infty}^{\infty} L_{n} e^{n z}-\frac{c}{24} .
$$

The PCFT formulation of the sine-Grodon model, suggests that the following formula should hold in the ultraviolet limit:

$$
\lim _{\ell \rightarrow 0}\left\{\left(\frac{\ell}{2 \pi}\right)^{2 \Delta_{\mathcal{O}}} \frac{\left\langle h_{1}, \ldots, h_{n}|\mathcal{O}(0)| h_{1}, \ldots h_{n}\right\rangle}{\left\langle h_{1}, \ldots, h_{n} \mid h_{1}, \ldots, h_{n}\right\rangle}\right\}=\frac{\left\langle\Delta\left|L_{n_{1}} \ldots L_{n_{j}} \mathcal{O}(0) L_{-n_{1}} \ldots L_{-n_{j}}\right| \Delta\right\rangle}{\left\langle\Delta\left|L_{n_{1}} \ldots L_{n_{j}} L_{-n_{1}} \ldots L_{-n_{j}}\right| \Delta\right\rangle},
$$

where $\Delta_{\mathcal{O}}$ is the scaling dimension of the local operator $\mathcal{O}$ in the complex Liouville CFT. In order to refer more easily to the soliton states, we introduce the notation:

$$
\left|I_{1}, \ldots, I_{n}\right\rangle=\left|h_{1}, \ldots, h_{n}\right\rangle,
$$

where $I_{j}$ s are the hole quantum numbers (3.8) in the NLIE (3.1). In this paper for our numerical checks we use the following pure hole states:

- $\left|-\frac{1}{2}, \frac{1}{2}\right\rangle \rightarrow|\Delta\rangle, \Delta=1-\frac{1}{\nu}$,

- $\left|-\frac{1}{2}, \frac{3}{2}\right\rangle \rightarrow|\Delta, \Delta+1\rangle, \Delta=1-\frac{1}{\nu}$,

- $\left|-\frac{3}{2}, \frac{3}{2}\right\rangle \rightarrow|\Delta+1, \Delta+1\rangle, \Delta=1-\frac{1}{\nu}$,

- $\left|-\frac{3}{2},-\frac{1}{2}, \frac{1}{2}, \frac{3}{2}\right\rangle \rightarrow|\Delta, \Delta\rangle, \Delta=1-\frac{2}{\nu}$,

where on the right hand side the (Liouville) CFT limit of the states are indicated, with the notation $|\Delta+1\rangle=L_{-1}|\Delta\rangle$. The set of operators we will consider is as follows:

$$
\mathcal{O}(0) \in\left\{\Phi_{2 \frac{1-\nu}{\nu}}, \Phi_{4 \frac{1-\nu}{\nu}}, \mathbf{l}_{-2} \Phi_{2 \frac{1-\nu}{\nu}}, \mathbf{l}_{-2} \cdot 1, \mathbf{l}_{-2}^{2} \cdot 1, \mathbf{l}_{-4} \cdot 1\right\} .
$$

The Liouville prediction for the case, when both the operator and the sandwiching state associated to some primary field is given by the formula (See section 6 of [13]):

$$
\mathcal{V}(\alpha, \kappa) \equiv \frac{\left\langle\Phi_{1-\kappa}(-\infty) \Phi_{\alpha+2 \frac{1-\nu}{\nu}}(0) \Phi_{1+\kappa}(\infty)\right\rangle}{\left\langle\Phi_{1-\kappa}(-\infty) \Phi_{\alpha}(0) \Phi_{1+\kappa}(\infty)\right\rangle}=\mu^{2} \Gamma(\nu)^{2} Y(x(\alpha)) W(\alpha, \kappa) W(\alpha,-\kappa),
$$

where $\mu$ and $x(\alpha)$ are defined in (5.11) and (5.7) respectively, and

$$
\begin{aligned}
Y(x) & =-2 \nu x \cdot \frac{\Gamma^{2}(\nu x+1 / 2-\nu / 2) \Gamma(\nu-2 \nu x)}{\Gamma^{2}(1 / 2+\nu / 2-\nu x) \Gamma(2 \nu x+1-\nu)} \cdot \frac{\Gamma(-2 \nu x)}{\Gamma(2 \nu x)}, \\
W(\alpha, \kappa) & =\frac{\Gamma(\alpha \nu / 2-\nu+1+\kappa \nu)}{\Gamma(-\alpha \nu / 2+\nu+\kappa \nu)} .
\end{aligned}
$$




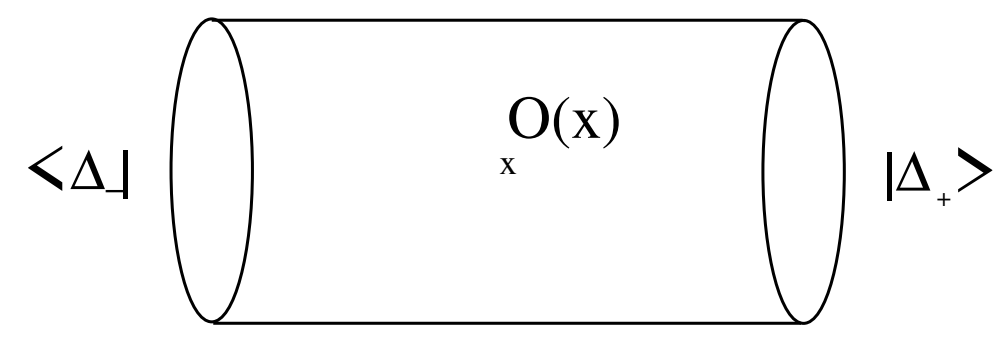

Figure 1. The pictorial representation of the cylindrical geometry for the small volume checks.

Using (7.5) it can be shown, that

$$
\frac{\left\langle\Phi_{1-\kappa}(-\infty) \Phi_{\alpha+4 \frac{1-\nu}{\nu}}(0) \Phi_{1+\kappa}(\infty)\right\rangle}{\left\langle\Phi_{1-\kappa}(-\infty) \Phi_{\alpha}(0) \Phi_{1+\kappa}(\infty)\right\rangle}=\mathcal{V}(\alpha, \kappa) \mathcal{V}\left(\alpha+2 \frac{1-\nu}{\nu}, \kappa\right) .
$$

With the help of the cylinder conformal Ward-identities, the 3-point functions of descendants can be related to those of the primaries [16, 45]. Again, for short we denote $|\Delta+1\rangle=L_{-1}|\Delta\rangle$. Then the following relations are obtained:

$$
\begin{aligned}
\frac{\left\langle\mathbf{l}_{-2} \Phi_{\alpha}\right\rangle_{\Delta}}{\left\langle\Phi_{\alpha}\right\rangle_{\Delta}}= & \Delta-\frac{c}{24}-\frac{\Delta_{\alpha}}{12} \\
\frac{\left\langle\mathbf{l}_{-4} \Phi_{\alpha}\right\rangle_{\Delta}}{\left\langle\Phi_{\alpha}\right\rangle_{\Delta}}= & \frac{\Delta_{\alpha}}{240} \\
\frac{\left\langle\mathbf{l}_{-2}^{2} \Phi_{\alpha}\right\rangle_{\Delta}}{\left\langle\Phi_{\alpha}\right\rangle_{\Delta}}= & \Delta^{2}-\Delta \frac{2 \Delta_{\alpha}+c+2}{12}+\frac{20 \Delta_{\alpha}^{2}+56 \Delta_{\alpha}+20 c \Delta_{\alpha}+5 c^{2}+22 c}{2880} . \\
\frac{\left\langle\Phi_{\alpha}\right\rangle_{\Delta+1}}{\left\langle\Phi_{\alpha}\right\rangle_{\Delta}}= & 2 \Delta+\Delta_{\alpha}^{2}-\Delta_{\alpha}, \\
\frac{\left\langle\mathbf{l}_{-2} \Phi_{\alpha}\right\rangle_{\Delta+1}}{\left\langle\Phi_{\alpha}\right\rangle_{\Delta}}= & 2 \Delta^{2}+\Delta \frac{12 \Delta_{\alpha}^{2}+34 \Delta_{\alpha}+24-c}{12}-\frac{\left(\Delta_{\alpha}-1\right) \Delta_{\alpha}\left(2 \Delta_{\alpha}-24+c\right)}{24} \\
\frac{\left\langle\mathbf{l}_{-4} \Phi_{\alpha}\right\rangle_{\Delta+1}}{\left\langle\Phi_{\alpha}\right\rangle_{\Delta}}= & \Delta \frac{241 \Delta_{\alpha}}{120}-\frac{\Delta_{\alpha}^{2}}{240}+\frac{\Delta_{\alpha}^{3}}{240}, \\
\frac{\left\langle\mathbf{l}_{-2}^{2} \Phi_{\alpha}\right\rangle_{\Delta+1}}{\left\langle\Phi_{\alpha}\right\rangle_{\Delta}}= & 2 \Delta^{3}+\Delta^{2} \frac{70-c+40 \Delta_{\alpha}+6 \Delta_{\alpha}^{2}}{6} \\
& +\Delta^{2} \frac{2400-218 c+5 c^{2}+4616 \Delta_{\alpha}-340 c \Delta_{\alpha}+1940 \Delta_{\alpha}^{2}-120 c \Delta_{\alpha}^{2}-240 \Delta_{\alpha}^{3}}{1440} \\
& +\frac{\left(\Delta_{\alpha}-1\right) \Delta_{\alpha}\left(2400-218 c+5 c^{2}-424 \Delta_{\alpha}+20 c \Delta_{\alpha}+20 \Delta_{\alpha}^{2}\right)}{2880} .
\end{aligned}
$$

Now, we are in the position to check whether the ultraviolet limit our formulas (4.15), (4.16) and (5.9) for the expectation values is described by the complex Liouville CFT. Unfortunately, we can not solve analytically the equations in the small volume limit, thus the comparison with CFT predictions, is performed through the numerical solution of the equations. In the rest of this section, we set $\mathcal{M}=1$ and measure everything in the units of soliton-mass. 


\subsection{The case of primaries $\Phi_{2 \frac{1-\nu}{\nu}}(0)$ and $\Phi_{4 \frac{1-\nu}{\nu}}(0)$}

The expectation values of these operators are given by the formulas (5.9):

$$
\begin{aligned}
\left\langle\Phi_{2 \frac{1-\nu}{\nu}}(0)\right\rangle= & C_{1}(0) \Pi(\nu)^{2-2 \nu}\left(\omega_{1,-1}(0)+\frac{i}{\nu} \cot \frac{\pi}{2 \nu}\right), \\
\left\langle\Phi_{4 \frac{1-\nu}{\nu}}(0)\right\rangle= & C_{2}(0) \Pi(\nu)^{8(1-\nu)}\left\{-\frac{1}{\nu^{2}} \cot \frac{\pi}{2 \nu} \cot \frac{3 \pi}{2 \nu}+\frac{i}{\nu} \cot \frac{\pi}{2 \nu} \omega_{3,-3}(0)+\frac{i}{\nu} \cot \frac{3 \pi}{2 \nu} \omega_{1,-1}(0)+\right. \\
& \left.\omega_{1,-1}(0) \omega_{3,-3}(0)-\omega_{1,-3}(0) \omega_{3,-1}(0)\right\} .
\end{aligned}
$$

Let us define the CFT limit of a finite volume expectation value by the formula:

$$
\langle\mathcal{O}(0)\rangle_{\mathrm{CFT}}=\lim _{\ell \rightarrow 0}\left\{\langle\mathcal{O}(0)\rangle\left(\frac{\ell}{2 \pi}\right)^{2 \Delta_{\mathcal{O}}}\right\} .
$$

Then for the four states we considered under (7.3), the CFT computations imply the following small volume limits for the primaries $\Phi_{2 m(1-\nu) / \nu}$ :

- $\left|-\frac{1}{2}, \frac{1}{2}\right\rangle$ :

$$
\left\langle\Phi_{2 m \frac{1-\nu}{\nu}}\right\rangle_{\mathrm{CFT}}=\left\langle\Phi_{2 m \frac{1-\nu}{\nu}}\right\rangle_{\Delta=1-\frac{1}{\nu}}
$$

- $\left|-\frac{3}{2},-\frac{1}{2}, \frac{1}{2}, \frac{3}{2}\right\rangle$ :

$$
\left\langle\Phi_{2 m \frac{1-\nu}{\nu}}\right\rangle_{\mathrm{CFT}}=\left\langle\Phi_{2 m \frac{1-\nu}{\nu}}\right\rangle_{\Delta=1-\frac{2}{\nu}}
$$

- $\left|-\frac{1}{2}, \frac{3}{2}\right\rangle$ :

$$
\left\langle\Phi_{2 m \frac{1-\nu}{\nu}}\right\rangle_{\mathrm{CFT}}=\left.\frac{2 \Delta+\Delta_{2 m(1-\nu) / \nu}^{2}-\Delta_{2 m(1-\nu) / \nu}}{2 \Delta}\left\langle\Phi_{2 m \frac{1-\nu}{\nu}}\right\rangle\right|_{\Delta=1-\frac{1}{\nu}},
$$

- $\left|-\frac{3}{2}, \frac{3}{2}\right\rangle$ :

$$
\left\langle\Phi_{2 m \frac{1-\nu}{\nu}}\right\rangle_{\mathrm{CFT}}=\left.\left(\frac{\left(2 \Delta+\Delta_{2 m(1-\nu) / \nu}^{2}-\Delta_{2 m(1-\nu) / \nu}\right)^{2}}{2 \Delta}\right)^{2}\left\langle\Phi_{2 m \frac{1-\nu}{\nu}}\right\rangle\right|_{\Delta=1-\frac{1}{\nu}}
$$

where according to (7.5):

$$
\left\langle\Phi_{2 m \frac{1-\nu}{\nu}}\right\rangle_{\Delta=1-\kappa}=\prod_{j=0}^{m-1} \nu\left(2 j \frac{1-\nu}{\nu}, \kappa\right) .
$$

The numerical results are obtained at $\nu=\frac{4}{7}$ are summarized in table 1 The CFT limit of the numerical results are taken from computing $\langle\mathcal{O}(0)\rangle\left(\frac{\ell}{2 \pi}\right)^{2 \Delta_{\mathcal{O}}}$ at $\ell=10^{-2}$. 


\begin{tabular}{|c|c|c|c|c|}
\hline & \multicolumn{2}{|c|}{$\left\langle\Phi_{2 \frac{1-\nu}{\nu}}\right\rangle_{\mathrm{CFT}}$} & \multicolumn{2}{c|}{$\left\langle\Phi_{4 \frac{1-\nu}{\nu}}\right\rangle_{\mathrm{CFT}}$} \\
\hline state & Numerics & CFT prediction & Numerics & CFT prediction \\
\hline$\left|-\frac{1}{2}, \frac{1}{2}\right\rangle$ & 2.7862399017 & 2.7862399479 & 0.7017902707 & 0.7017903948 \\
\hline$\left|-\frac{3}{2},-\frac{1}{2}, \frac{1}{2}, \frac{3}{2}\right\rangle$ & 2.3026776339 & 2.3026776429 & 1.5315414850 & 1.5315415383 \\
\hline$\left|-\frac{1}{2}, \frac{3}{2}\right\rangle$ & 3.3651982417 & 3.3651988981 & 0.4830504382 & 0.4830505315 \\
\hline$\left|-\frac{3}{2}, \frac{3}{2}\right\rangle$ & 4.0644590918 & 4.0644610068 & 0.3324887760 & 0.3324893268 \\
\hline
\end{tabular}

Table 1. Numerical data for the expectation values of primaries $\Phi_{2 m(1-\nu) \nu}(0)$, with $m=1,2$, at the $\left(\nu=\frac{4}{7}, \ell=10^{-2}\right)$ point of the parameter space.

\subsection{Expectation values of descendant fields}

In order to consider expectation values of descendant fields, one needs to know how the fermionic basis in (5.2) is related to the Virasoro basis used in the Liouville 3-point functions. For our studies we need to know this relation up to some low lying levels. The relation is known up to level 8 [45]. Nevertheless, here we summarize from [13], only the most important formulas, we need.

The fermionic basis can be expressed in terms of the Virasoro descendants according to formula:

$$
\beta_{I^{+}}^{*} \gamma_{I^{-}}^{*} \Phi_{\alpha}(0)=\prod_{2 j-1 \in I^{+}} D_{2 j-1}(\alpha) \prod_{2 j-1 \in I^{-}} D_{2 j-1}(2-\alpha)\left[P_{I^{+}, I^{-}}^{\mathrm{even}}+d_{\alpha} P_{I^{+}, I^{-}}^{\text {odd }}\right]
$$

where

$$
\begin{aligned}
d_{\alpha} & =\frac{\nu(\nu-2)}{\nu-1}(\alpha-1), \\
D_{2 j-1}(\alpha) & =-\sqrt{\frac{i}{\nu}} \Gamma(\nu)^{-\frac{2 j-1}{\nu}}(1-\nu)^{\frac{2 j-1}{2}} \frac{\Gamma\left(\frac{\alpha}{2}+\frac{1}{2 \nu}(2 j-1)\right)}{(j-1) ! \Gamma\left(\frac{\alpha}{2}+\frac{1-\nu}{2 \nu}(2 j-1)\right)},
\end{aligned}
$$

and $P_{I^{+}, I^{-}}^{\text {even }}$ and $P_{I^{+}, I^{-}}^{\text {odd }}$ are polynomial expressions of the even Virasoro generators $\left\{\mathbf{1}_{-2 k}\right\}$. For the first few levels they take the form as follows [13]:

$$
\begin{array}{ll}
P_{\{1\},\{1\}}^{\text {even }}=\mathbf{l}_{-2}, & P_{\{1\},\{1\}}^{\text {odd }}=0, \\
P_{\{1\},\{3\}}^{\text {even }}=P_{\{3\},\{1\}}^{\text {even }}=\frac{1}{2} \mathbf{l}_{-2}^{2}+\frac{c-16}{9} \mathbf{l}_{-4}, & P_{\{1\},\{3\}}^{\text {odd }}=P_{\{3\},\{1\}}^{\text {odd }}=-\frac{1}{3} \mathbf{l}_{-4} .
\end{array}
$$

The descendants of the "shifted-primaries" $\Phi_{\alpha+2 n \frac{1-\nu}{\nu}}(0)$ can be computed from the formula (5.12).

\subsubsection{The case of $\left\langle 1_{-2} \Phi_{2 \frac{1-\nu}{\nu}}\right\rangle$}

Using (5.12), (7.17) and (5.1) one obtains the following expression for $\left\langle\mathbf{1}_{-2} \Phi_{2 \frac{1-\nu}{\nu}}\right\rangle$ :

$$
\left\langle\mathbf{1}_{-2} \Phi_{2 \frac{1-\nu}{\nu}}\right\rangle=\frac{C_{1}(0) \frac{1}{i \nu} \cot \frac{\pi}{2 \nu} \Pi(\nu)^{4-2 \nu}}{D_{1}\left(2 \frac{1-\nu}{\nu}\right) D_{1}\left(2-2 \frac{1-\nu}{\nu}\right)} \omega_{3,-1}(0) .
$$




\begin{tabular}{|c|c|c|c|c|}
\hline & \multicolumn{2}{|c|}{$\left\langle\mathbf{1}_{-2} \Phi_{2 \frac{1-\nu}{\nu}}\right\rangle_{\mathrm{CFT}}$} & \multicolumn{2}{c|}{$\omega_{1,1}(0)_{\mathrm{CFT}}$} \\
\hline state & Numerics & CFT prediction & Numerics & CFT prediction \\
\hline$\left|-\frac{1}{2}, \frac{1}{2}\right\rangle$ & 1.5423829631 & 1.5423828283 & 1.2069251545 & 1.2069249851 \\
\hline$\left|-\frac{3}{2},-\frac{1}{2}, \frac{1}{2}, \frac{3}{2}\right\rangle$ & 5.3043825168 & 5.3043824274 & $5.1062212309 \mathrm{i}$ & $5.1062210908 \mathrm{i}$ \\
\hline$\left|-\frac{1}{2}, \frac{3}{2}\right\rangle$ & 4.4320078359 & 4.432008303 & $3.4350943930 \mathrm{i}$ & $3.4350941884 \mathrm{i}$ \\
\hline$\left|-\frac{3}{2}, \frac{3}{2}\right\rangle$ & 5.3529430027 & 5.3529450933 & $3.4350944355 \mathrm{i}$ & $3.4350941884 \mathrm{i}$ \\
\hline
\end{tabular}

Table 2. Numerical data for the expectation values of the level 2 descendant fields; $\mathbf{l}_{-2} \Phi_{2(1-\nu) / \nu}$ and $\mathbf{l}_{-2} 1$, at the $\left(\nu=\frac{4}{7}, \ell=10^{-2}\right)$ point of the parameter space.

Then for the four states under consideration the Liouville CFT gives the following predictions:

- $\left|-\frac{1}{2}, \frac{1}{2}\right\rangle$ :

$$
\left\langle\mathbf{1}_{-2} \Phi_{2 \frac{1-\nu}{\nu}}\right\rangle_{\mathrm{CFT}}=\left.\left(\Delta-\frac{c}{24}-\frac{\Delta_{2(1-\nu) / \nu}}{12}\right)\left\langle\Phi_{2 \frac{1-\nu}{\nu}}\right\rangle\right|_{\Delta=1-\frac{1}{\nu}}
$$

- $\left|-\frac{3}{2},-\frac{1}{2}, \frac{1}{2}, \frac{3}{2}\right\rangle$ :

$$
\left\langle\mathbf{1}_{-2} \Phi_{2 \frac{1-\nu}{\nu}}\right\rangle_{\mathrm{CFT}}=\left.\left(\Delta-\frac{c}{24}-\frac{\Delta_{2(1-\nu) / \nu}}{12}\right)\left\langle\Phi_{2 \frac{1-\nu}{\nu}}\right\rangle\right|_{\Delta=1-\frac{2}{\nu}}
$$

- $\left|-\frac{1}{2}, \frac{3}{2}\right\rangle$ :

$$
\left\langle\mathbf{1}_{-2} \Phi_{2 \frac{1-\nu}{\nu}}\right\rangle_{\mathrm{CFT}}=\left.\frac{F_{-2}\left(\Delta, \Delta_{2(1-\nu) / \nu}\right)}{2 \Delta}\left\langle\Phi_{2 \frac{1-\nu}{\nu}}\right\rangle\right|_{\Delta=1-\frac{1}{\nu}},
$$

- $\left|-\frac{3}{2}, \frac{3}{2}\right\rangle$ :

$$
\left\langle\mathbf{1}_{-2} \Phi_{2 \frac{1-\nu}{\nu}}\right\rangle_{\mathrm{CFT}}=\left.\frac{2 \Delta+\Delta_{2(1-\nu) / \nu}^{2}-\Delta_{2(1-\nu) / \nu}}{(2 \Delta)^{2}} F_{-2}\left(\Delta, \Delta_{2(1-\nu) / \nu}\right)\left\langle\Phi_{2 \frac{1-\nu}{\nu}}\right\rangle\right|_{\Delta=1-\frac{1}{\nu}}
$$

where we introduced the function from the right hand side of second line of (7.9):

$$
F_{-2}\left(\Delta, \Delta_{\alpha}\right)=2 \Delta^{2}+\Delta \frac{12 \Delta_{\alpha}^{2}+34 \Delta_{\alpha}+24-c}{12}-\frac{\left(\Delta_{\alpha}-1\right) \Delta_{\alpha}\left(2 \Delta_{\alpha}-24+c\right)}{24} .
$$

The corresponding numerical results obtained at $\ell=10^{-2}$ and $\nu=\frac{4}{7}$, can be found in table 2 . 


\subsubsection{Expectation values of the descendants of the unity}

Now, instead of expressing the expectation values of the operators under consideration, we express the $\omega_{i j}(\alpha)$ matrix elements in terms of the expectation values of the low lying descendants of the primary field $\Phi_{\alpha}(0)$. The formulas one obtains are as follows:

$$
\begin{aligned}
& \omega_{1,1}(\alpha)=\mu^{-2 / \nu} D_{1}(\alpha) D_{1}(2-\alpha) \frac{\left\langle\mathbf{1}_{-2} \Phi_{\alpha}(0)\right\rangle}{\left\langle\Phi_{\alpha}(0)\right\rangle} \\
& \omega_{3,1}(\alpha)=\mu^{-4 / \nu} D_{3}(\alpha) D_{1}(2-\alpha)\left\{\frac{1}{2} \frac{\left\langle\mathbf{l}_{-2}^{2} \Phi_{\alpha}(0)\right\rangle}{\left\langle\Phi_{\alpha}(0)\right\rangle}+\left(\frac{c-16}{9}+\frac{d_{\alpha}}{3}\right) \frac{\left\langle\mathbf{1}_{-4} \Phi_{\alpha}(0)\right\rangle}{\left\langle\Phi_{\alpha}(0)\right\rangle}\right\}, \\
& \omega_{1,3}(\alpha)=\mu^{-4 / \nu} D_{1}(\alpha) D_{3}(2-\alpha)\left\{\frac{1}{2} \frac{\left\langle\mathbf{l}_{-2}^{2} \Phi_{\alpha}(0)\right\rangle}{\left\langle\Phi_{\alpha}(0)\right\rangle}+\left(\frac{c-16}{9}-\frac{d_{\alpha}}{3}\right) \frac{\left\langle\mathbf{1}_{-4} \Phi_{\alpha}(0)\right\rangle}{\left\langle\Phi_{\alpha}(0)\right\rangle}\right\} .
\end{aligned}
$$

For the comparison of numerical and the CFT results, we define the CFT limit of the above $\omega$ s as follows:

$$
\omega_{j k}(\alpha)_{\mathrm{CFT}}=\lim _{\ell \rightarrow 0}\left\{\omega_{j k}(\alpha)\left(\frac{\ell}{2 \pi}\right)^{\operatorname{dim} \omega_{j k}(\alpha)}\right\},
$$

where $\operatorname{dim} \omega_{j k}(\alpha)$ is the length dimension of $\omega_{j k}(\alpha)$. For the 3 special cases in (7.26) $\operatorname{dim} \omega_{j k}(\alpha)=j+k$. We check (7.26) in the $\alpha \rightarrow 0$ limit. As a consequence of (7.8) and (7.9), in this case in the small volume limit $\left\langle\mathbf{1}_{-4} 1\right\rangle=0$. Thus, $\omega_{1,3}(0)_{\mathrm{CFT}}=\omega_{3,1}(0)_{\mathrm{CFT}}$.

Using the formulas (7.8) and (7.9), for the four specific states, one obtains the CFT predictions as follows:

- $\left|-\frac{1}{2}, \frac{1}{2}\right\rangle$ :

$$
\omega_{1,1}(0)_{\mathrm{CFT}}=\left.\Pi(\nu)^{-2} D_{1}(0) D_{1}(2)\left(\Delta-\frac{c}{24}\right)\right|_{\Delta=1-\frac{1}{\nu}},
$$

- $\left|-\frac{3}{2},-\frac{1}{2}, \frac{1}{2}, \frac{3}{2}\right\rangle$ :

$$
\omega_{1,1}(0)_{\mathrm{CFT}}=\left.\Pi(\nu)^{-2} D_{1}(0) D_{1}(2)\left(\Delta-\frac{c}{24}\right)\right|_{\Delta=1-\frac{2}{\nu}},
$$

- $\left|-\frac{1}{2}, \frac{3}{2}\right\rangle$ :

$$
\omega_{1,1}(0)_{\mathrm{CFT}}=\left.\Pi(\nu)^{-2} D_{1}(0) D_{1}(2) \frac{F_{-2}(\Delta, 0)}{2 \Delta}\right|_{\Delta=1-\frac{1}{\nu}}
$$

- $\left|-\frac{3}{2}, \frac{3}{2}\right\rangle$ :

$$
\omega_{1,1}(0)_{\mathrm{CFT}}=\left.\Pi(\nu)^{-2} D_{1}(0) D_{1}(2) \frac{F_{-2}(\Delta, 0)}{2 \Delta}\right|_{\Delta=1-\frac{1}{\nu}} .
$$




\begin{tabular}{|c|c|c|c|c|}
\hline & \multicolumn{2}{|c|}{$\omega_{1,3}(0)_{\mathrm{CFT}}$} & $\omega_{3,1}(0)_{\mathrm{CFT}}$ & \\
\hline state & Numerics & CFT prediction & Numerics & CFT prediction \\
\hline$\left|-\frac{1}{2}, \frac{1}{2}\right\rangle$ & $1.9427100198 \mathrm{i}$ & $1.9427092046 \mathrm{i}$ & $1.9427100198 \mathrm{i}$ & $1.9427092046 \mathrm{i}$ \\
\hline$\left|-\frac{3}{2},-\frac{1}{2}, \frac{1}{2}, \frac{3}{2}\right\rangle$ & $47.1269102712 \mathrm{i}$ & $47.1269074676 \mathrm{i}$ & $47.1269102712 \mathrm{i}$ & $47.1269074676 \mathrm{i}$ \\
\hline$\left|-\frac{1}{2}, \frac{3}{2}\right\rangle$ & $35.7155944324 \mathrm{i}$ & $35.7155920899 \mathrm{i}$ & $35.7155944324 \mathrm{i}$ & $35.7155920899 \mathrm{i}$ \\
\hline$\left|-\frac{3}{2}, \frac{3}{2}\right\rangle$ & $35.7155949192 \mathrm{i}$ & $35.7155920899 \mathrm{i}$ & $35.7155949192 \mathrm{i}$ & $35.7155920899 \mathrm{i}$ \\
\hline
\end{tabular}

Table 3. Numerical data for the expectation values of the level 4 descendants of the unity, at the $\left(\nu=\frac{4}{7}, \ell=10^{-2}\right)$ point of the parameter space.

Before turning to the case of $\omega_{3,1}(0)_{\mathrm{CFT}}$, from (7.8) and (7.9) taken at $\Delta_{\alpha}=0$, it is worth to introduce the functions as follows:

$$
\begin{aligned}
& F_{0}(\Delta)=\left\langle\mathbf{l}_{-2}^{2} \cdot 1\right\rangle_{\Delta}=\Delta^{2}-\Delta \frac{c+2}{12}+\frac{5 c^{2}+22 c}{2880} \\
& F_{1}(\Delta)=\frac{\left\langle\mathbf{l}_{-2}^{2} \cdot 1\right\rangle_{\Delta+1}}{\langle 1\rangle_{\Delta}}=2 \Delta^{3}+\Delta^{2} \frac{70-c}{6}+\Delta \frac{5 c^{2}-218 c+2400}{1440} .
\end{aligned}
$$

Then one obtains the following results in the CFT limit:

- $\left|-\frac{1}{2}, \frac{1}{2}\right\rangle$ :

$$
\omega_{3,1}(0)_{\mathrm{CFT}}=\left.\Pi(\nu)^{-4} D_{3}(0) D_{1}(2) \frac{1}{2} F_{0}(\Delta)\right|_{\Delta=1-\frac{1}{\nu}},
$$

- $\left|-\frac{3}{2},-\frac{1}{2}, \frac{1}{2}, \frac{3}{2}\right\rangle$ :

$$
\omega_{3,1}(0)_{\mathrm{CFT}}=\left.\Pi(\nu)^{-4} D_{3}(0) D_{1}(2) \frac{1}{2} F_{0}(\Delta)\right|_{\Delta=1-\frac{2}{\nu}},
$$

- $\left|-\frac{1}{2}, \frac{3}{2}\right\rangle$ :

$$
\omega_{3,1}(0)_{\mathrm{CFT}}=\left.\Pi(\nu)^{-4} D_{3}(0) D_{1}(2) \frac{F_{1}(\Delta)}{4 \Delta}\right|_{\Delta=1-\frac{1}{\nu}},
$$

- $\left|-\frac{3}{2}, \frac{3}{2}\right\rangle$ :

$$
\omega_{3,1}(0)_{\mathrm{CFT}}=\left.\Pi(\nu)^{-4} D_{3}(0) D_{1}(2) \frac{F_{1}(\Delta)}{4 \Delta}\right|_{\Delta=1-\frac{1}{\nu}} .
$$

The corresponding numerical results obtained at $\ell=10^{-2}$ and $\nu=\frac{4}{7}$, can be found in tables 2 and 3. 


\section{Summary and conclusions}

In the fundamental paper [13], 1-point functions or in other words ground state expectation values of exponential fields and of their Virasoro descendants have been determined in the sine-Gordon model defined in cylindrical geometry. The derivation was done in the socalled fermionic basis discovered in the integrable light-cone lattice regularization [30] of the model. ${ }^{7}$ The relation of the fermionic basis of operators to that of the Virasoro basis, has been determined up to level 8 [45]. The main advantage of the fermionic basis is that in this basis, the 1-point functions can be given by simple determinant expressions of a single nontrivial matrix $\omega_{j k}(\alpha)$, whose matrix elements can be obtained by solving linear integral equations containing the counting-function of the ground state as a fundamental building block of its kernel.

In this paper, we extended these results from ground state expectation values to expectation values in any eigenstate of the Hamiltonian of the sine-Gordon model. Structurally all formulas of [13] remain the same, but in the case of expectation values in excited states the matrix $\omega_{j k}(\alpha)$ will depend on the sandwiching state. Thus, the linear equations, which determine the matrix $\omega_{j k}(\alpha)$, are needed to be modified by appropriate source terms, which characterize the sandwiching state.

Having these equations at hand, we performed several tests on the equations in the pure multi-soliton sector. In the large volume limit we performed three tests. We have shown, that the excited state formulation of the matrix $\omega_{j k}(\alpha)$ still respects the nonlinear compatibility equation, which is necessary to get unique answer for each expectation value of each operator. We also checked, that our excited state formulation of the expectation values is consistent with the previous LeClair-Mussardo type large volume series conjecture of [24]. Finally, from the multi-soliton expectation values of the set of primaries $\Phi_{2 n(1-\nu) / \nu}$, $n \in \mathbb{N}$, we computed the classical limit of connected diagonal form-factors up to two soliton states, and compared them to the results of semi-classical computations coming from direct field theoretical computations of ref. [44]. The comparison gave perfect agreement.

The equations were checked in the ultraviolet limit, as well. In this limit, the expectation values tend to 3-point functions of the complex Liouville conformal field theory. While, in the large volume limit the equations determining the expectation values, can be solved analytically, in the ultraviolet limit they can be solved only numerically. Nevertheless, the accurate solution of the equations allowed us to check our results for various operators and low lying multi-soliton excitations, against Liouville 3-point functions. Perfect agreement was found in this regime, too.

The sine-Gordon was the first integrable model with non-diagonally scattering theory, where all finite volume diagonal matrix elements of local operators could have been determined. The volume dependence in the final formulas for the expectation values is encoded into the volume dependence of the counting-function. This function satisfies certain nonlinear integral equations and governs the finite volume dependence of the energy of the sandwiching state. This description of the finite volume spectrum is very specific for the sine-Gordon model and there is no general method to find an analogue of this description

\footnotetext{
${ }^{7}$ Namely, in the 6-vertex model with appropriate alternating inhomogeneities.
} 
to other models. For some specific examples see references [49-52] etc. Nevertheless, there is another method to describe the finite volume spectrum in integrable quantum field theories with non-diagonally scattering theory. This is Thermodynamic Bethe Ansatz (TBA) method, where the rapidity dependent pseudo-energies satisfy the nonlinear TBA integral equations and govern the volume dependence of the energies. Though the concrete form of the TBA equations are more complicated than that of the counting function, this formulation has the advantage, that it can be generalized to any non-diagonally scattering theory. Thus, rephrasing the results of this paper in the language of the TBA pseudoenergies, could give a deeper insight into the structure of the formulas and help in finding a generalization to other important models.

\section{Acknowledgments}

The author would like to thank Zoltán Bajnok and Fedor Smirnov for useful discussions. This work was supported by the NKFIH research Grant K116505 and by a HungarianFrench bilateral exchange project.

Open Access. This article is distributed under the terms of the Creative Commons Attribution License (CC-BY 4.0), which permits any use, distribution and reproduction in any medium, provided the original author(s) and source are credited.

\section{References}

[1] Z. Bajnok and R.A. Janik, String field theory vertex from integrability, JHEP 04 (2015) 042 [arXiv: 1501.04533] [INSPIRE].

[2] Z. Bajnok, R.A. Janik and A. Wereszczyński, HHL correlators, orbit averaging and form factors, JHEP 09 (2014) 050 [arXiv: 1404.4556] [INSPIRE].

[3] L. Hollo, Y. Jiang and A. Petrovskii, Diagonal Form Factors and Heavy-Heavy-Light Three-Point Functions at Weak Coupling, JHEP 09 (2015) 125 [arXiv:1504.07133] [INSPIRE].

[4] Y. Jiang and A. Petrovskii, Diagonal form factors and hexagon form factors, JHEP 07 (2016) 120 [arXiv: 1511.06199] [INSPIRE].

[5] Y. Jiang, Diagonal Form Factors and Hexagon Form Factors II. Non-BPS Light Operator, JHEP 01 (2017) 021 [arXiv:1601.06926] [INSPIRE].

[6] F.H.L. Essler and R.M. Konik, Applications of massive integrable quantum field theories to problems in condensed matter physics, in From Fields to Strings: Circumnavigating Theoretical Physics, M. Shifman, A. Vainshtein and J. Wheater eds., World Scientific (2005), pp. 684-830 [cond-mat/0412421].

[7] B. Pozsgay and G. Takács, Form-factors in finite volume I: Form-factor bootstrap and truncated conformal space, Nucl. Phys. B 788 (2008) 167 [arXiv:0706.1445] [INSPIRE].

[8] B. Pozsgay and G. Takács, Form factors in finite volume. II. Disconnected terms and finite temperature correlators, Nucl. Phys. B 788 (2008) 209 [arXiv:0706.3605] [InSPIRE].

[9] B. Pozsgay, Lüscher's mu-term and finite volume bootstrap principle for scattering states and form factors, Nucl. Phys. B 802 (2008) 435 [arXiv:0803.4445] [INSPIRE]. 
[10] Z. Bajnok, J. Balog, M. Lájer and C. Wu, Field theoretical derivation of Lüscher's formula and calculation of finite volume form factors, JHEP 07 (2018) 174 [arXiv: 1802.04021] [INSPIRE].

[11] Z. Bajnok, M. Lájer, B. Szepfalvi and I. Vona, Leading exponential finite size corrections for non-diagonal form factors, JHEP 07 (2019) 173 [arXiv:1904.00492] [INSPIRE].

[12] H. Boos, M. Jimbo, T. Miwa and F. Smirnov, Hidden Grassmann Structure in the XXZ Model IV: CFT limit, Commun. Math. Phys. 299 (2010) 825 [arXiv:0911.3731] [InSPIRE].

[13] M. Jimbo, T. Miwa and F. Smirnov, Hidden Grassmann structure in the XXZ model V: sine-Gordon model, Lett. Math. Phys. 96 (2011) 325 [arXiv:1007.0556] [InSPIRE].

[14] C. Babenko and F. Smirnov, One point functions of fermionic operators in the Super Sine Gordon model, Nucl. Phys. B 946 (2019) 114698 [arXiv:1905.09602] [INSPIRE].

[15] S. Negro and F. Smirnov, On one-point functions for sinh-Gordon model at finite temperature, Nucl. Phys. B 875 (2013) 166 [arXiv:1306.1476] [InSPIRE].

[16] Z. Bajnok and F. Smirnov, Diagonal finite volume matrix elements in the sinh-Gordon model, Nucl. Phys. B 945 (2019) 114664 [arXiv:1903.06990] [INSPIRE].

[17] Z. Bajnok and C. Wu, Diagonal form factors from non-diagonal ones, in 2017 MATRIX Annals, Springer, Cham Switzerland (2019), pp. 141-151 [arXiv:1707.08027] [INSPIRE].

[18] T. Pálmai and G. Takács, Diagonal multisoliton matrix elements in finite volume, Phys. Rev. D 87 (2013) 045010 [arXiv: 1209.6034] [INSPIRE].

[19] A. Leclair and G. Mussardo, Finite temperature correlation functions in integrable QFT, Nucl. Phys. B 552 (1999) 624 [hep-th/9902075] [INSPIRE].

[20] B. Pozsgay, Form factor approach to diagonal finite volume matrix elements in Integrable QFT, JHEP 07 (2013) 157 [arXiv: 1305.3373] [INSPIRE].

[21] B. Pozsgay, I.M. Szécsényi and G. Takács, Exact finite volume expectation values of local operators in excited states, JHEP 04 (2015) 023 [arXiv:1412.8436] [INSPIRE].

[22] H. Saleur, A Comment on finite temperature correlations in integrable QFT, Nucl. Phys. B 567 (2000) 602 [hep-th/9909019] [INSPIRE].

[23] B. Pozsgay, Mean values of local operators in highly excited Bethe states, J. Stat. Mech. 1101 (2011) P01011 [arXiv:1009.4662] [InSPIRE].

[24] Á. Hegedüs, Lattice approach to finite volume form-factors of the Massive Thirring (sine-Gordon) model, JHEP 08 (2017) 059 [arXiv:1705.00319] [INSPIRE].

[25] Á. Hegedüs, Exact finite volume expectation values of $\bar{\Psi} \Psi$ in the massive Thirring model from light-cone lattice correlators, JHEP 03 (2018) 047 [arXiv: 1710.09583] [INSPIRE].

[26] T.T. Truong and K.D. Schotte, Quantum Inverse Scattering Method And The Diagonal To Diagonal Transfer Matrix Of Vertex Models, Nucl. Phys. B 220 (1983) 77 [InSPIRE].

[27] T.T. Truong and K.D. Schotte, The Quantum Field Theories Associated With A 'Staggered' Ice Type Model, Nucl. Phys. B 230 (1984) 1 [InSPIRE].

[28] T.T. Truong, On soluble cases of staggered ice-rule on a square lattice, in Non-Linear Equations in Classical and Quantum Field Theory, N. Sanchez ed., Springer, Lect. Notes Phys. 226 (1985) 234.

[29] M.F. Weiss and K.D. Schotte, Lattice Approach To The Spectrum Of The Massive Thirring Model, Nucl. Phys. B 225 (1983) 247 [INSPIRE]. 
[30] C. Destri and H.J. de Vega, Light Cone Lattice Approach to Fermionic Theories in 2D: The Massive Thirring Model, Nucl. Phys. B 290 (1987) 363 [InSPIRE].

[31] A. Klümper, M.T. Batchelor and P.A. Pearce, Central charges of the 6- and 19-vertex models with twisted boundary conditions, J. Phys. A 24 (1991) 3111 [InSPIRE].

[32] C. Destri and H.J. de Vega, New thermodynamic Bethe ansatz equations without strings, Phys. Rev. Lett. 69 (1992) 2313 [INSPIRE].

[33] C. Destri and H.J. de Vega, New approach to thermal Bethe ansatz, hep-th/9203064 [INSPIRE].

[34] C. Destri and H.J. De Vega, Unified approach to thermodynamic Bethe Ansatz and finite size corrections for lattice models and field theories, Nucl. Phys. B 438 (1995) 413 [hep-th/9407117] [INSPIRE].

[35] D. Fioravanti, A. Mariottini, E. Quattrini and F. Ravanini, Excited state Destri- De Vega equation for sine-Gordon and restricted sine-Gordon models, Phys. Lett. B 390 (1997) 243 [hep-th/9608091] [INSPIRE].

[36] C. Destri and H.J. de Vega, Nonlinear integral equation and excited states scaling functions in the sine-Gordon model, Nucl. Phys. B 504 (1997) 621 [hep-th/9701107] [InSPIRE].

[37] G. Feverati, F. Ravanini and G. Takács, Nonlinear integral equation and finite volume spectrum of sine-Gordon theory, Nucl. Phys. B 540 (1999) 543 [hep-th/9805117] [INSPIRE].

[38] G. Feverati, F. Ravanini and G. Takács, Truncated conformal space at $c=1$, nonlinear integral equation and quantization rules for multi-soliton states, Phys. Lett. B 430 (1998) 264 [hep-th/9803104] [INSPIRE].

[39] G. Feverati, F. Ravanini and G. Takács, Scaling functions in the odd charge sector of sine-Gordon/massive Thirring theory, Phys. Lett. B 444 (1998) 442 [hep-th/9807160] [INSPIRE].

[40] G. Feverati, Finite volume spectrum of sine-Gordon model and its restrictions, Ph.D. Thesis, University of Bologna, Bologna Italy (2000) [hep-th/0001172] [INSPIRE].

[41] D. Fioravanti and M. Rossi, A Braided Yang-Baxter algebra in a theory of two coupled lattice quantum KdV: Algebraic properties and ABA representations, J. Phys. A 35 (2002) 3647 [hep-th/0104002] [INSPIRE].

[42] D. Fioravanti and M. Rossi, Exact conserved quantities on the cylinder. 1: Conformal case, JHEP 07 (2003) 031 [hep-th/0211094] [INSPIRE].

[43] D. Fioravanti and M. Rossi, Exact conserved quantities on the cylinder. 2: Off critical case, JHEP 08 (2003) 042 [hep-th/0302220] [INSPIRE].

[44] Z. Bajnok and R.A. Janik, Classical limit of diagonal form factors and HHL correlators, JHEP 01 (2017) 063 [arXiv: 1607.02830] [INSPIRE].

[45] H. Boos, Fermionic basis in conformal field theory and thermodynamic Bethe ansatz for excited states, SIGMA 7 (2011) 007 [arXiv: 1010.0858] [INSPIRE].

[46] H. Boos and F. Smirnov, New results on integrable structure of conformal field theory, J. Phys. A 51 (2018) 374003 [arXiv:1610.09537] [INSPIRE].

[47] V.S. Dotsenko and V.A. Fateev, Conformal Algebra and Multipoint Correlation Functions in Two-Dimensional Statistical Models, Nucl. Phys. B 240 (1984) 312 [INSPIRE].

[48] A.B. Zamolodchikov, Mass scale in the sine-Gordon model and its reductions, Int. J. Mod. Phys. A 10 (1995) 1125 [inSPIRE]. 
[49] J. Balog and Á. Hegedűs, $A d S_{5} \times S^{5}$ mirror TBA equations from $Y$-system and discontinuity relations, JHEP 08 (2011) 095 [arXiv: 1104.4054] [INSPIRE].

[50] Á. Hegedüs, Finite size effects in the SS model: Two component nonlinear integral equations, Nucl. Phys. B 679 (2004) 545 [hep-th/0310051] [INSPIRE].

[51] V. Kazakov and S. Leurent, Finite size spectrum of $\mathrm{SU}(N)$ principal chiral field from discrete Hirota dynamics, Nucl. Phys. B 902 (2016) 354 [arXiv: 1007.1770] [INSPIRE].

[52] N. Gromov, V. Kazakov and P. Vieira, Finite Volume Spectrum of $2 D$ Field Theories from Hirota Dynamics, JHEP 12 (2009) 060 [arXiv:0812.5091] [INSPIRE].

[53] S.R. Coleman, The Quantum sine-Gordon Equation as the Massive Thirring Model, Phys. Rev. D 11 (1975) 2088 [INSPIRE].

[54] T.R. Klassen and E. Melzer, Sine-Gordon not equal to massive Thirring and related heresies, Int. J. Mod. Phys. A 8 (1993) 4131 [hep-th/9206114] [INSPIRE]. 